Supporting Information

\title{
Molecular Origin of Mechanical Sensitivity of the Reaction Rate in Anthracene Cyclophane Isomerization Reveals Structural Motifs for Rational Design of Mechanophores
}

\author{
Nikolay V. Plotnikov and Todd J. Martinez \\ nplotnikov@gmail.com; todd.martinez@stanford.edu
}

\section{Additional Computational Details on Methods Available experimental data}

The dependence of the rate constant on pressure for the PI to BA reverse isomerization was estimated ${ }^{1}$ from two experiments. The first experiment established the dependence of the rate constant on pressure at a fixed temperature from a biexponential fit of the kinetic data (Table S1 and Table 1, Eq. 1. in ref 1). The second experiment established the dependence of the rate constant on temperature at a fixed pressure. The first experiment was used to estimate the activation volume, while the second experiment was used to assess the activation energy of the reaction.

Changes in the reaction rate constant are related to the changes in the activation free energy by the transition state theory, Eq. 1 of the main text:

$$
\ln k=\ln \frac{\kappa}{\beta h}-\beta \Delta g^{\neq}
$$

Thus, we can estimate the change in the activation free energy $\Delta \Delta \mathrm{g}^{\neq}$, from the rate constant $\left(k_{o}\right)$ and the activation free energies $\left(\Delta g^{\ddagger}\right)$ under standard conditions and the rate constant under different conditions $k(T, p)$ :

$$
\Delta \Delta g^{\neq}=\beta^{-1} \ln \left(\frac{k(p, T)}{k_{0}}\right)
$$

We convert a set of rate constants determined by fitting the kinetic data under different pressures to the pressure-induced free energy changes (Table S1).

Furthermore, the activation free energy can be represented with a sum of three contributions: the internal energy, the entropic effect and the mechanical components: $\Delta g^{\neq}=\Delta U^{\neq}-T \Delta S^{\neq}+p \Delta V^{\neq}$

That is:

$$
\ln k(\beta, p=\text { const })=\left[\ln \frac{\kappa}{\beta h}+\frac{\Delta S^{\neq}}{k_{B}}\right]-\beta\left(\Delta U^{\neq}+p \Delta V^{\neq}\right)
$$

The temperature dependence of the rate constant gives the well-known Arrhenius type linear dependence of $\ln k$ on the inverse temperature $\beta$, with the slope providing an estimate for the activation enthalpy, $\Delta H^{\neq}=\Delta U^{\neq}+p \Delta V^{\neq}$

The activation enthalpy was estimated to be $\sim 7 \mathrm{~kJ} / \mathrm{mol}(\sim 2 \mathrm{kcal} / \mathrm{mol})$ at $0.9 \mathrm{GPa}$ (in the temperature range $314^{-}$ $296 \mathrm{~K})$ and $\sim 93 \mathrm{~kJ} / \mathrm{mol}(\sim 22 \mathrm{kcal} / \mathrm{mol})$ at normal pressure (in the temperature range $298-339 \mathrm{~K})$. 
Similarly, for a given temperature, assuming the independence of the activation entropy, the activation (internal) energy and the activation volume on pressure, one expects a linear dependence of the $\ln k$ on pressure, with the linear slope providing an estimate for the activation volume:

$$
\ln k(\beta=\text { const }, p)=\left(\ln \frac{\kappa}{\beta h}+\beta T \Delta S^{\neq}-\beta \Delta U^{\neq}\right)-p\left(\beta \Delta V^{\neq}\right)
$$

Experimentally ${ }^{1}$ determined slope corresponds to the activation volume of -16 $\AA^{3}$ in the pressure range of o-1.5 GPa (Figure 5 of ref. 1).

\section{Potential Energy Surfaces from Unrestricted Density Functional Theory}

The choice of UB3LYP functional is based on the comparison of several DFT functionals with available experimental data for the studied system: activation energy, reaction heat, and $R_{1}$ and $R_{2}$ values in equilibrium structures for the final states (Table $S_{2}$ ). Potential energy scans along $R_{1}$ and $R_{2}$ values were used to estimate those values theoretically (Figures $\left.S_{2}-S_{5}\right)$. Theoretically predicted values for $R_{1}$ and $R_{2}\left(C_{9}-C_{9}\right.$, and $C_{10}-C_{10}$ interatomic distances in Figure 1) in bis-anthracene (BA) and its photoisomer (PI) were generally close to the x-ray values of 2.76 $\AA$ for BA and 1.65 $\AA$ for PI. ${ }^{2}$ The closest structural agreement was observed for B3LYP functional with both 6$31 \mathrm{G}$ and $6-31 \mathrm{G}^{*}$ basis sets: 2.80 and 1.675 respectively (accuracy limited by $0.025 \AA$ grid spacing). The PBE functional predicts slightly elongated bond lengths (in $\AA$ ) in the PI: 1.700 and 2.800 for BA, while BLYP predicts overstretched bonds in both final states: 1.725 and 2.825, respectively. Experimental estimates for the activation energy vary from $22.5^{1} \mathrm{kcal} / \mathrm{mol}$ to $22.3^{3}-23.9^{4} \mathrm{kcal} / \mathrm{mol}$ and are about $-8.5 \mathrm{kcal} / \mathrm{mol}$ for the reaction heat. The agreement of computed thermochemical parameters with these experimental values is very poor for BLYP functional, and is much better for PBE and the hybrid B3LYP functional (particularly with 6-31 $\mathrm{G}^{*}$ basis set). The latter provides the closest estimate to the structural and thermochemical experimental values, even compared to more expensive functionals ${ }^{5}$.

Obtaining the unrestricted spin symmetry broken wavefunction in the Self-Consistent Field (SCF) approach, particularly near the stability limit (where the unrestricted and restricted solutions are close in energy), requires generating a symmetry broken initial guess and using appropriate SCF algorithm to ensure convergence to the desired solution. In order to obtain a continuous potential energy surface and to find the spin symmetry broken solution in QChem ${ }^{6}$, we added 40-50 \% Lowest Unoccupied Molecular Orbital (LUMO) to the Highest Occupied Molecular Orbital (HOMO), in addition in some cases we used two separate DIIS error vectors. For calculations performed with TeraChem ${ }^{7}$ we rotated alpha- LUMO and HOMO by 40-45 degrees and used a combination of DIIS $^{8}$ and ADIIS 9 algorithms, since DIIS sometimes favors the spin symmetric solution at the stability limit. On the other hand, for the studied system we observed that the DIIS usually converges in a fewer iterations, while the ADIIS is more robust. That is, we continuously monitored the change in energy during SCF. If DIIS led to the energy increase in 2 consequent iterations, we switched to ADIIS and iterated SCF until we saw the energy decrease for 2 iterations in a row, in which case we switched back to DIIS. This procedure was repeated (one more time) if the DIIS led to the increase in energy again. In rare cases, when this approach failed we switched to ADIIS to reach the convergence.

The reaction potential energy surfaces were computed using BLYP, PBE and B3LYP DFT-functionals with an empirical dispersion correction ${ }^{10}$ in the following way. Starting from the photoisomer optimized geometry we first compute the concerted path by increasing $R_{1}$ and $R_{2}$ simultaneously by $0.025 \AA$. Next, starting from the optimized geometries (representing the concerted path) we carried out the potential energy scan in perpendicular directions, that is by increasing $R_{1}$ and decreasing $R_{2}$ in $0.025 \AA$ increments. During minimization a special care was taken to ensure the spin-symmetry breaking of the wavefunction, which resulted in a lower energy, spin-contaminated solutions. Continuity of the potential energy surface was monitored in parallel with continuity of the spin-square expectation values.

Starting from the PI both $\mathrm{R}_{1}$ and $\mathrm{R}_{2}$ distances were simultaneously constrained to the values from 1.600 to 2.850 $\AA$ with a $0.025 \AA$ interval. The potential energy scan was performed sequentially using the geometries and the molecular orbitals coefficients as an initial guess from the previous step with QChem 4.2. Next, starting from the optimized geometries for the concerted path, the potential energy scan was performed in perpendicular directions, i.e. by increasing one bond by $0.025 \AA$ and decreasing another bond by $0.025 \AA$. This showed that the sequential reaction path is preferential and the corresponding transition state is lower in energy. Potential energy surfaces with corresponding spin-square expectation value $<\mathrm{S}^{2}>$ are given in Figures $\mathrm{S}_{2}-\mathrm{S}_{5}$ and in Table $\mathrm{S}_{2}$. Figure $S_{3}$ depicts $<S^{2}>$ values for $U_{3} L Y P-D_{2} / / 6-31 G$ reaction potential energy surface given in the main text and for 
BLYP//6-31G, PBE//6-31G and B3LYP//6-31G* model chemistries, respectively. Figures $\mathrm{S}_{4}$ depicts the concerted pathway and projections of the TS region on the dividing surface (and the corresponding $\left\langle\mathrm{S}^{2}\right\rangle$ values).

The key thermodynamic parameters for the minimum energy path are given in Table 1 for UB3LYP-D2//6-31G. The energy barrier is $17.4 \mathrm{kcal} / \mathrm{mol}$ and the reaction energy change is $\mathbf{- 2 2 . 5} \mathrm{kcal} / \mathrm{mol}$. We approximated the free energy surface in vacuum by the corresponding potential energy surface with entropic terms evaluated using the harmonic approximation. Note, that the difference between the corresponding internal and free energies of activation and of reaction is less than $1 \mathrm{kcal} / \mathrm{mol}$. (See Table 1 ). The entropic and zero-point energy corrections were computed for critical points on the reaction path in vacuum for the minimum energy geometries. The total zero-point energy correction is $-2.0 \mathrm{kcal} / \mathrm{mol}$ to the activation free energy barrier and $0.2 \mathrm{kcal} / \mathrm{mol}$ to the reaction energy change, while the entropic correction is $-0.3 \mathrm{kcal} / \mathrm{mol}$ and $-0.4 \mathrm{kcal} / \mathrm{mol}$, respectively. Thus, in the harmonic approximation, under standard conditions the entropic effects on the reaction is minor. The basis set effect on the potential energy surface was also estimated using the $6-31 \mathrm{G}^{*}$ basis set. While the polarized basis set indeed improved the agreement with experimentally available thermochemical parameters, it has a small effect on the structural parameters as well as on the reaction path.

The minimum energy reaction path in vacuum was computed using the Nudged Elastic Band (NEB) method ${ }^{11}$ as implemented in TeraChem ${ }^{12,13}$. This was accomplished by using the final states and the transition state from the constrained energy minimization and constructing the initial molecular path of 6o NEB images totally by extrapolation. Separately, the NEB path made of 24 images was used for additional points to estimate the pressure dependence of the free energy surface. The projection of the NEB path on the $2 \mathrm{D}$ potential energy map is shown in the top right panel of Figure 4. The corresponding surface of the expectation values of the spin square operator $\left\langle\mathrm{S}^{2}\right\rangle$ is provided below. The spin square expectation value and the energy along the reaction coordinate are continuous, which indicates that the lowest energy, symmetry-broken solutions were successfully found and also shows the spin. Spin contamination (predominantly by the triplet, $\left\langle S^{2}\right\rangle=3$ ) was observed only in the TS region past the dividing surface towards PS, thus, demonstrating the diradical character of the TS. The final states were not spin-contaminated in DFT. The minimum energy path corresponds to the stepwise mechanism in agreement with a previous DFT study. ${ }^{5}$

\section{Reaction Path under Pressure}

While the error in the absolute barrier height can be quite substantial and significantly varies between functionals, the error in estimating the barrier reduction and its variance between functionals is significantly smaller ( Figure S6). That is, while the difference in the absolute activation energy varies among functionals, the difference in the effect of pressure on both the activation and reaction free energies is notably smaller and is rather consistent among different functionals. That is, the reduction in the free energy barrier for the concerted path was found to be $-11.0,-11.1$ and $-10.4 \mathrm{kcal} / \mathrm{mol}$ under 2.67 GPa for BLYP, PBE and B3LYP functionals. The corresponding change in the reaction free energy was computed to be -11.4, -12.0 and -12.3. This clearly indicates the error cancellation. Of course, at larger pressures the agreement worsened for BLYP which severely underestimated the original barrier height. At 5.3 GPa the effect of pressure on the concerted barrier with PBE and B3LYP functionals, were in close agreement: -15.4 and $-14.6 \mathrm{kcal} / \mathrm{mol}$ as well as the effect on the reaction free energy change: -13.5 and $-15.0 \mathrm{kcal} / \mathrm{mol}$, respectively (Figure $\mathrm{S6}$ ).

A set of $R_{1}$ and $R_{2}$ values from the gas-phase NEB path was used to approximate the minimum free energy path under pressure, since a minor shift in the transverse direction to the reaction coordinate would result in a low error due to flattening of the TS region (Figure $S_{7}$ ). This was achieved by constraining the corresponding $R_{1}$ and $R_{2}$ values by adding two harmonic potentials with the force constants of $400 \mathrm{kcal} /\left(\mathrm{mol} \cdot \AA^{2}\right)$ and propagating a set of QM MD-trajectories for 6-10 ps (using 2 fs time steps, what requires an increased 2 a.u. mass for hydrogen atoms) under different pressures controlled with our implementation of the ideal gas pressure bath. Note that all other degrees of freedom were unconstrained. The experiment in ref. ${ }^{1}$ was reportedly carried out under hydrostatic pressure conditions. In our simulations we reproduced the hydrostatic pressure using the ideal gas pressure bath coupled with $a b$ initio MD. From sets of MD trajectories we computed the effect for 8 different pressures in the range 0.1-7.3 GPa (o.1 GPa; 0.6 GPa; 0.9 GPa; 1.4 GPa; 2.0 GPa; $2.7 \mathrm{GPa}$; $5.3 \mathrm{GPa}$; 7.3 GPa) on the reaction free energy surfaces. The free energy surfaces are defined in $R_{1}$ and $R_{2}$ coordinates, with a linear combination of those: $R_{1}+R_{2}$ for the diagonal projection and $R_{1}-R_{2}$ for the perpendicular projection, or with the NEB bead corresponding to a particular set of $R_{1}$ and $R_{2}$. The diagonal projection is a convenient $1 \mathrm{D}$ coordinate, which shows evolution along the reaction path, and the perpendicular projection shows the curvature of TS region, projected on the dividing surface. Initially we performed the mapping of the free energy surface in a way 
similar to the potential energy surface scan - that is by constraining $R_{1}$ and $R_{2}$ values with harmonic potentials, and by scanning first along the concerted path and then by scanning in the perpendicular direction from the highest point in free energy at the concerted path. From these experiments we first computed the free energy profile along the concerted path for UB3LYP (see Figure S6 A) and for PBE (see Figure S6 B).

The data from these trajectories was combined (after discarding first 1ooo steps for equilibration) using a set of the free energy estimators described elsewhere ${ }^{14}$. The standard deviation between free energy estimators (BAR, FEP, LRA/TDI and WHAM) was used to estimate the error in the computed free energy shifts. The free energy profiles were obtained by reweighting with WHAM and with the umbrella sampling/free energy perturbation protocols. The deviation between the free energy shifts and the free energy profiles were small and systematic along the reaction path, and the free energy shifts were used as estimates of the minimum free energy reaction path (NEB paths). SCF energies computed for configurations from these trajectories were used to estimate the internal energies by the umbrella sampling reweighting procedure, where only configurations with the bias less than $1.5 \mathrm{kT}$ were considered. Next, these configurations were also used to compute the quasi-harmonic entropy. Errors in our estimates of the internal energy, the entropic contributions and the mechanical contribution are naturally higher than those in the free energy by about an order of magnitude, and are within $1-2 \mathrm{kcal} / \mathrm{mol}$. The error of $1 \mathrm{kcal} / \mathrm{mol}$ will cause different error in the volume estimates, with a significant error at small pressures and small errors at high pressures. We examined the error for the internal energy profile by performing the betaspline fit and adjusting the smoothing parameter to ensure smoothness and continuity of the profile. The average deviations from the spline fits was below $1 \mathrm{kcal} / \mathrm{mol}(\sim 1.5 \mathrm{kT}$ at $\mathrm{T}=300 \mathrm{~K})$. The entropic profiles were assumed to be a straight line - that is independent on the reaction coordinate (Figure S14). The corresponding standard deviation from the mean for the entropy profiles were: $0.7 \mathrm{kcal} / \mathrm{mol}(7.3 \mathrm{GPa})$; 0.6 (5.3 GPa); 0.8 (2.7 GPa) and 1.0 $\mathrm{kcal} / \mathrm{mol}$ ( $0.9 \mathrm{GPa})$.

2D free energy surfaces are computed in the following way: first, we computed only the concerted path by adding two harmonic potentials with the force constants of $400 \mathrm{kcal} /\left(\mathrm{mol} \cdot \AA^{2}\right), R_{1}$ and $R_{2}$ are constrained to the same values, with spacing of $0.025 \AA$ A between 1.6 and $2.85 \AA$ A. From the concerted path we also determined the effect of pressure on the reaction free energy change. Next, by constraining $R_{1}$ and $R_{2}$ to the values corresponding to the TS determined from the energy minimization, we computed the stepwise TS region of the free energy surface and position it (relative to the reactants) using the free energy shifts corresponding to the concerted pathway. The free energy regions in between the paths were sampled using potentials with smaller force constants and a sparse grid in $R_{1}$ and $R_{2}$ spacing. These trajectories were combined using WHAM protocol similar to wham-2.0.8 ${ }^{15}$.

While all values in Table 1 of the main text are given relative to values for the corresponding RS, Figure S8 depicts the internal energy profiles relative to the energy of the equilibrium PS in vacuum. One can see that in the pressure range 0.1-1.4 GPa the internal energy profiles at 30oK are shifted up by the same amount relative to the minimum energy path in the gas phase (which corresponds to o K). Note that no zero-point energy correction is included. At pressure of $2.0 \mathrm{GPa}$ and higher the RS energy is destabilized increasingly more relative to the PS.

\section{The ideal gas pressure bath}

The effect of pressure in $a b$ initio MD was simulated using our adaptation of the statistical ideal gas pressure bath $^{16,17}$ which represents the effect of the mechanical stress (hydrostatic pressure). The simulation system, treated quantum mechanically (QM region), is immersed in the reservoir of particles, which obey the ideal gas statistics (i.e. particle fluctuation in a volume element, the equation of state and the velocity distribution). These particles don't interact with each other but do interact with the QM region via the repulsive term of the LennardJones potential. While, in general, in our implementation we closely followed the original computational protocol ${ }^{17}$ in a few instances we introduced some changes. The main change, which significantly simplifies the original implementation, is the use of a single box by removing the momentum for the center of mass, thus preventing translational motion of the QM system.

First, we use a single imaginary box, which contains a large number of the "ideal gas" atoms and the simulation system. The size of the imaginary box is one of the parameters, which can be used for controlling the pressure. The cubic box side length $L$ is set to be:

(S5) $\quad L=r_{\max }[i]-r_{\min }[i]$

where 


$$
\begin{aligned}
& r_{\min }[i]=r_{c o m}[i]-r_{\max Q M}-6 n_{R C U T} \\
& r_{\max }[i]=r_{c o m}[i]+r_{\max Q M}+6 n_{R C U T}
\end{aligned}
$$

here $r_{\text {com }}[i]$ are the coordinates of the center of mass of the molecule (QM region), $r_{\max Q M}$ is the farthest coordinate of QM region from the center of mass, $n_{R C U T}$ is the number of cut-off radii. The cutoff radius was taken to be $6 \AA$ which is $2 \sigma$ of the Lennard-Jones potential for the ideal gas atoms ${ }^{17}$.

Initially, the box (excluding the inner QM compartment) is filled by generating a random distribution of $N$ particles (computed from the ideal gas equation of state):

$$
p\left(V-V_{Q M}\right)=N k T
$$

Velocity components $(\mathrm{x}, \mathrm{y}, \mathrm{z})$ of these particles are generated according to the Maxwell distribution:

$$
p(v)=\frac{1}{\sqrt{2 \pi \sigma^{2}}} \exp \left(-\frac{v^{2}}{2 \sigma^{2}}\right)
$$

Where $\sigma^{2}=k_{B} T / m_{i}$

\section{Pressure control}

The pressure is raised incrementally by increasing the average number of particles, $n_{i n}$, injected through each side of the box with surface area of $A_{b o x}=L^{2}$ per time step $\tau=\Delta t$ :

$$
p=\frac{6 n_{i n} \sqrt{2 \pi T k_{B} m_{a u x}}}{A_{b o x} \tau}
$$

For a given temperature $\mathrm{T}$, time step $\mathrm{t}$ and mass of the ideal gas particles $n_{i n}$ and $A_{b o x}$ are the variables adjusted to reach the desired pressure with $n_{\text {in }}$ also being an integer.

Thus, one way of implementation involves defining an average number of particles injected through each side of the box and adjusting the surface area according to eq. (S10). That is:

$$
\delta L=\sqrt{\frac{A_{b o x}}{6}}-L_{b o x}
$$

$$
r_{\max }[i]=r_{\max }[i]+0.5 \delta L \text { and } r_{\min }[i]=r_{\min }[i]-0.5 \delta L \text { however one has to make sure that }
$$

$$
0.5 \delta L<5 n_{R C U T}
$$

The number of injected particles $k$ is drawn from the Poisson distribution for each side at each MD step (that's why it could be rather advantageous to have $\mathrm{k}>1$, we chose $\mathrm{k}=4$ ):

$$
p(k)=\frac{n_{i n}^{k}}{k !} \exp \left(-n_{i n}\right)
$$

For $k \in[0 ; \infty)$

The components of velocity perpendicular to the side are generated using the Rayleigh distribution:

(S13) $\quad p(v)=\frac{v}{\sigma^{2}} \exp \left(-\frac{v^{2}}{2 \sigma^{2}}\right)$

while other components are generated by the Maxwell distribution. Until the desired pressure is reached and the equilibrium between leaving and entering particles is established, the QM system is frozen. After the box is pressurized (i.e. $p \cong p_{T G T}$ at $n_{i n}=n_{T G T}$ ) the total center of mass momentum is set to zero. Next, at each MD 
step we follow the Verlet algorithm. That is, for each Cartesian component we propagate the classical equaition of motion. For velocities:

(S14) $\quad \dot{x}\left(t+\frac{1}{2} \Delta t\right)=\dot{x}(t)+\frac{1}{2} \Delta t \cdot \ddot{x}(t)$

For positions:

(S15) $x(t+\Delta t)=x(t)+\Delta t \cdot \dot{x}\left(t+\frac{1}{2} \Delta t\right)$

Next, we update the list of the ideal gas particles to remove those, which left the box. Next, we generate a number of new particles using eqs. (S12) at each face of the cube with their positions being randomly distributed over its surface and with the velocity components generated with eqs. ( $\left.\mathrm{S}_{9}\right)$ and $\left(\mathrm{S}_{13}\right)$. Then, we propagate the newly inserted particles:

$$
x(t+\Delta t)=x(t)+U(0,1) \Delta t \cdot \dot{x}\left(t+\frac{1}{2} \Delta t\right)
$$

where $U(0,1)$ is the uniform distribution and discard the particles which left the box.

Next, we compute acceleration using QM derivatives for all QM atoms:

(S17) $\ddot{x}(t+\Delta t)=-\frac{1}{m} \frac{\partial}{\partial x} E(x(t+\Delta t))$

Finally, we update velocities for all atoms in the cube:

$$
\dot{x}(t+\Delta t)=\dot{x}\left(t+\frac{1}{2} \Delta t\right)+\frac{1}{2} \Delta t \cdot \ddot{x}(t+\Delta t)
$$

The Rayleigh distribution and the Maxwell distribution are generated following ref. ${ }^{18}$; the random number generator, the Poisson and the uniform distributions were taken from ref. ${ }^{19}$

The instantaneous pressure in the box is computed from the virial theorem ${ }^{20}$ :

$$
p_{\text {in }}=\frac{1}{3 L^{3}}\left(E_{k i n}+\sum_{i} \mathbf{r} \cdot \mathbf{F}\right)
$$

The average pressure is obtained by averaging the instantaneous pressure over MD trajectory.

In case of Lennard-Jones pair-wise potential between particles $i$ and $j$ separated by $r$ and interacting via force $f_{i j}$ , eq. (S19) becomes:

$$
p_{i n}=\frac{1}{V}\left(N k T+\frac{1}{3} \sum_{i} \sum_{j>i} r_{i j} \cdot f_{i j}\right)
$$

\section{Effect of mechanical stress from constrained minimization}

After we determined the main deformational mode caused by pressure, we used the following approach to estimate effect of a particular deformation. The effect of a directional mechanical stress (anisotropic compression) was estimated from the constrained energy minimization using four C-C distances (two such distances are denoted by $\boldsymbol{Z}$ in Figure 1 of the main text while other two are the symmetric distances on the opposite side of the molecule). By starting from geometries for the RS, TS and PS which were determined from the computed reaction potential energy surfaces, further scans were performed (keeping $R_{1}$ and $R_{2}$ fixed) by decreasing $Z$ distances in $0.025 \AA$ increments until they become less than or equal to $R_{1}$ and $R_{2}$. We performed numerical differentiation of the energy vs. deformation energy curves and thus obtained energy vs. energyderivative (the negative force) curves. This technique is used by a number of other workers. ${ }^{21,22}$ 
By performing adiabatic compression of molecules, we computed the increase of the internal energy in response to deformation $d z$. At the equilibrium the applied external force is counter balanced by the internal force, therefore the force balance is attained:

$$
\text { (S21) } \quad f_{\text {ext }}=f_{\text {int }}
$$

Where

$$
\text { (S22) } f_{\text {int }}=-\left(\frac{\partial U}{\partial z}\right)
$$

At infinitely small increments $d z$ the derivative can be approximated by finite increments, and obtained from the numerical differentiation of the potential energy curves. Next, we fitted the energy versus deformation profiles with polynomials and differentiated them, thus obtaining how internal energy of a molecule changes in response to the applied force.

Attractive interactions between two aromatic systems (which is generally called $\pi$ - $\pi$ stacking) can be considered in terms of the electrostatic interactions between electronic densities of two stacked rings and the dispersion interactions. ${ }^{23}$ The charge distribution from RS through TS to PS undergoes relatively small changes in vacuum, what is expected for the reaction in nonpolar environment. Compression of the critical points also doesn't lead to the noticeable changes in the charge distribution with RMSD and maximum deviation (from the corresponding equilibrium geometry in vacuum) being 0.029 and 0.073 (RS); 0.006 and 0.016 (TS) and 0.005 and 0.012 (PS). In all states, the offset $\pi$-stacked geometry minimizes electrostatic repulsion between $\pi$-clouds and favors the attractive electrostatic interactions with the positive periphery (Figure $\mathrm{S}_{15}$ top panel). Thus a shear stress bringing two rings in the eclipse orientation will increase the energy of all states due to electrostatic repulsion between same charges. Decreasing the $Z$ interatomic distances will lead to the short-range VdW repulsive interactions becoming the dominant term.

We removed rotational and translational degrees of freedom and superimposed averaged structures at simulated pressures (Figures S9-S11).

The green curve in Figure $S_{15}$ shows that the anisotropic compression (defined with Z-coordinate in Figure 1) leads to the predominant destabilization of the reactants by up to $15 \mathrm{kcal} / \mathrm{mol}$.

\section{Tables}

Table S1 Experimentally estimated effect of pressure on the rate constants (from Ref. ${ }^{1}$ ) and the corresponding activation free energy changes.

\begin{tabular}{lll}
\hline \hline $\mathrm{P}, \mathrm{GPa}$ & $\mathrm{k}, \mathrm{min}^{-1}$ & ${ }^{\mathrm{a}} \Delta \Delta \mathrm{g}^{\ddagger}, \mathrm{kcal} / \mathrm{mol}$ \\
\hline $\mathrm{o}$ & 0.0003 & $\mathrm{o}$ \\
0.62 & 0.0012 & 0.82 \\
0.71 & 0.0089 & 2.01 \\
0.85 & 0.0053 & 1.70 \\
0.93 & 0.0343 & $\mathrm{~b}$ \\
1.12 & 0.0142 & 2.29 \\
1.40 & 0.0604 & 3.15 \\
1.95 & 0.0788 & 3.30 \\
2.56 & 0.1035 & 3.47 \\
\hline \hline
\end{tabular}

${ }^{\mathrm{a}} \Delta \Delta g^{*}=0.593 \ln \left(\frac{k_{i}}{k_{0}}\right) ;{ }^{\mathrm{b}}$ Estimate from the Arrhenius plot (lnk vs $\left.1 / \mathrm{T}\right)$ for the barrier reduction is $20.8 \mathrm{kcal} / \mathrm{mol}$ at $\sim 0.9 \mathrm{GPa}$; Also note that $1 \mathrm{GPa}=0.14393 \mathrm{kcal} /\left(\mathrm{mol} \AA^{3}\right)$, which allows to convert $\Delta \mathrm{V}^{\ddagger}$ to $\Delta \Delta \mathrm{g}^{\ddagger}$. 
Table S2 A. Energetics of the concerted and sequential pathways for the PI to BA conversion in vacuum from the energy minimization. The entries for each functional correspond to reactants, concerted TS, sequential TS, and products from top to bottom. Energies are given relative to the corresponding reactants state energies.

\begin{tabular}{|c|c|c|c|}
\hline & $E, \mathrm{kcal} / \mathrm{mol}$ & $R_{1} / / R_{2}, \AA$ & $<S^{2}>, \AA$ \\
\hline \multirow[t]{4}{*}{ UBLYP-D2//6-31G } & 0.0 & $1.725 / / 1.725$ & 0.00 \\
\hline & 16.0 & $2.225 / / 2.225$ & 0.73 \\
\hline & 13.9 & $2.550 / / 1.800$ & 0.71 \\
\hline & -29.2 & $2.825 / / 2.825$ & 0.00 \\
\hline \multirow[t]{4}{*}{ UPBE-D2//6-31G } & 0.0 & $1.700 / / 1.700$ & 0.0 \\
\hline & 21.7 & $2.225 / / 2.225$ & 0.76 \\
\hline & 18.7 & $2.550 / / 1.800$ & 0.75 \\
\hline & -19.0 & $2.800 / / 2.800$ & 0.0 \\
\hline \multirow[t]{4}{*}{$\mathrm{UB}_{3} \mathrm{LYP}-\mathrm{D}_{2} / / 6-31 \mathrm{G}$} & 0.0 & $1.675 / / 1.675$ & 0.0 \\
\hline & 23.7 & $2.175 / / 2.175$ & 0.63 \\
\hline & $17 \cdot 7$ & $2.475 / / 1.725$ & 0.76 \\
\hline & -22.5 & $2.800 / / 2.800$ & 0.0 \\
\hline \multirow[t]{4}{*}{$\mathrm{UB}_{3} \mathrm{LYP}-\mathrm{D}_{2} / / 6-31 \mathrm{G}^{*}$} & 0.0 & $1.675 / / 1.675$ & 0.0 \\
\hline & 26.1 & $2.175 / / 2.175$ & 0.53 \\
\hline & 19.7 & $2.525 / / 1.725$ & 0.85 \\
\hline & $-17 \cdot 4$ & $2.800 / / 2.800$ & 0.0 \\
\hline \multicolumn{4}{|l|}{ From ref ${ }^{5}$} \\
\hline \multirow[t]{4}{*}{ UMo6-2x//6-31 $G^{* *}$} & 0.0 & $1.645 / / 1.645$ & \\
\hline & 38.9 & $2.200 / / 2.200$ & \\
\hline & 27.8 & $2.626 / / 1.692$ & \\
\hline & -9.1 & $2.802 / / 2.802$ & \\
\hline \multirow[t]{4}{*}{ UB97D//6-31+G* } & 0.0 & $1.688 / / 1.688$ & \\
\hline & 17.9 & $2.200 / / 2.200$ & \\
\hline & 15.6 & $2.609 / / 1.814$ & \\
\hline & -21.2 & $2.823 / / 2.823$ & \\
\hline \multirow[t]{3}{*}{ Experiment } & 0.0 & $1.65 / / 1.65^{2}$ & \\
\hline & $22.5^{1} ; 22.3^{3} ; 23.9^{4}$ & & \\
\hline & $-8.5^{4}$ & $2.76 / / 2.76^{2}$ & \\
\hline
\end{tabular}




\section{Figures}

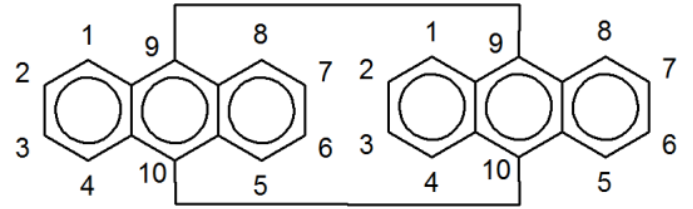

Figure S1 Connectivity and numeration of carbon atoms in the anthracene moieties of bis-anthracene. $\mathrm{R}_{1}$ and $\mathrm{R}_{2}$ interatomic distances between $C_{9}$ and $C_{10}$ atoms of the top and of the bottom anthracene fragments are used to define the reaction coordinate. 

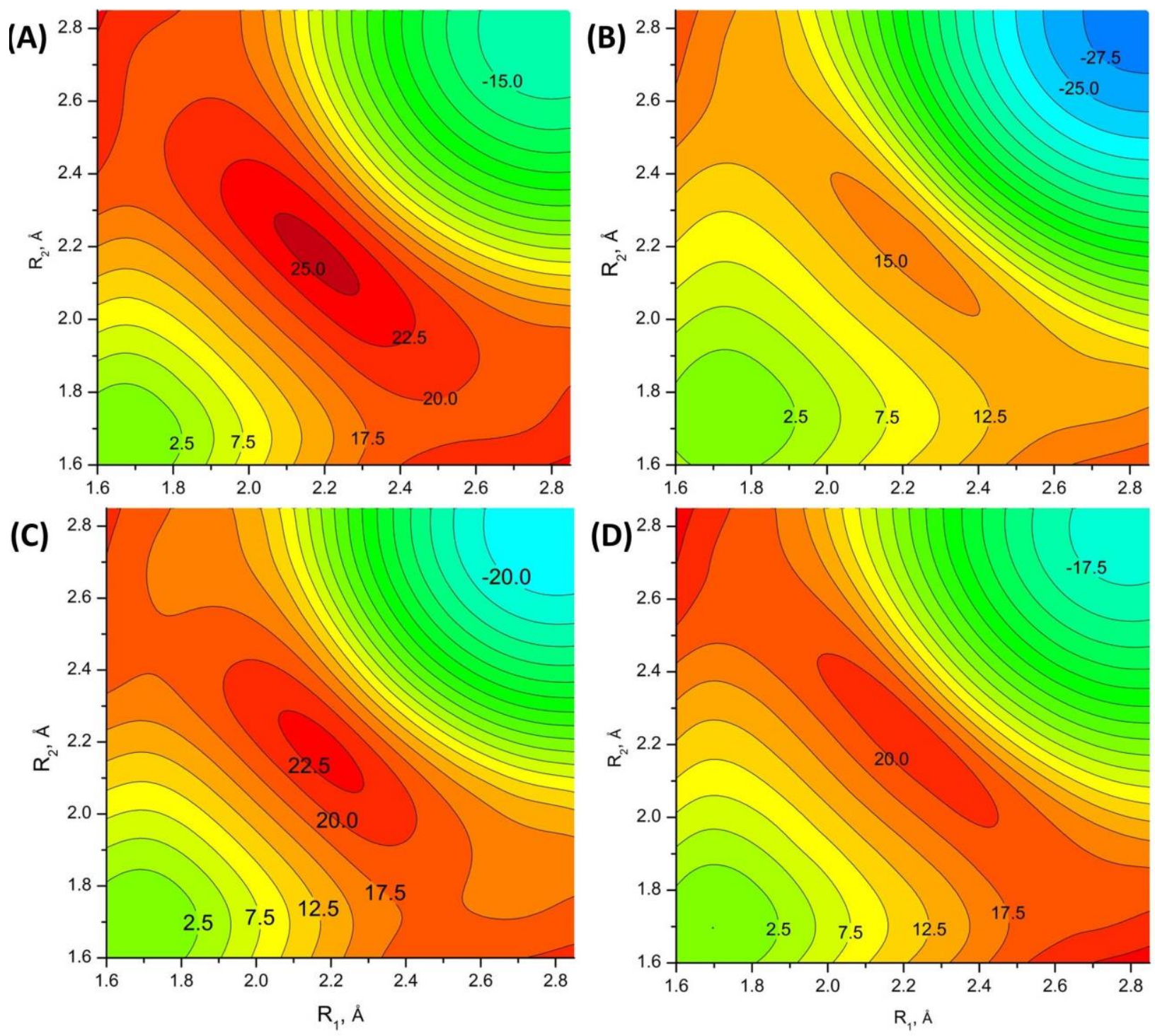

Figure S2 Several potential energy surfaces with different density functionals were examined to select the one that is closest to the experimental data for the activation energy $(22-24 \mathrm{kcal} / \mathrm{mol})$, for the reaction heat (8-10 $\mathrm{kcal} / \mathrm{mol})$, as well as for structural data for $\mathrm{C}_{9}-\mathrm{C}_{10}$ bond lengths. All functionals include Grimme's empirical dispersion correction and correspond to the spin-unrestricted solutions of: (A) UB3LYP//6-31G(d); (B) UBLYP//6-31G; (C) UB3LYP//6-31G; (D) UPBE//6-31G. 
(A)

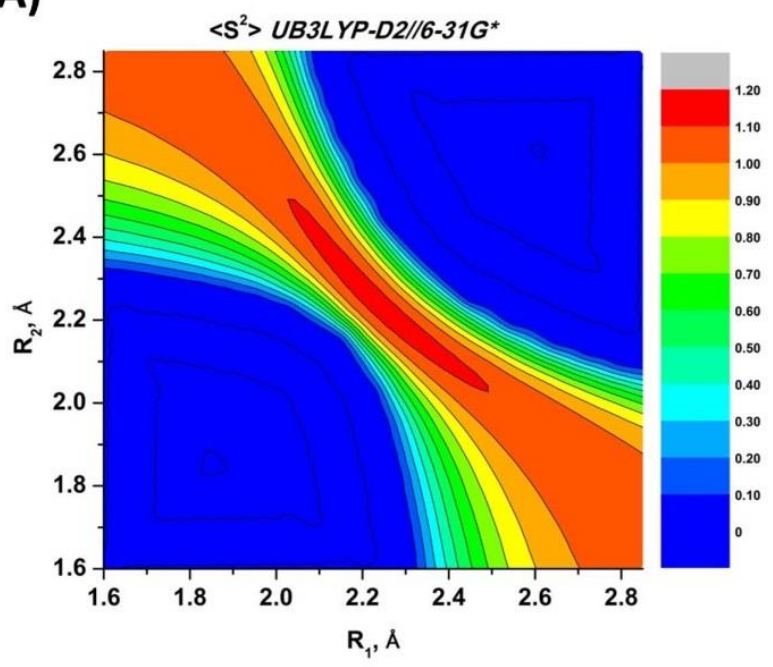

(C)

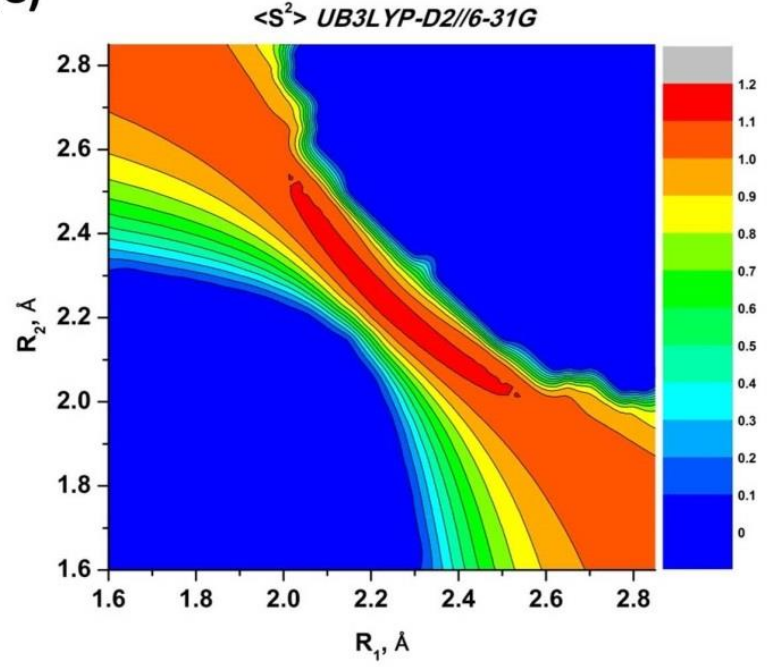

(B)

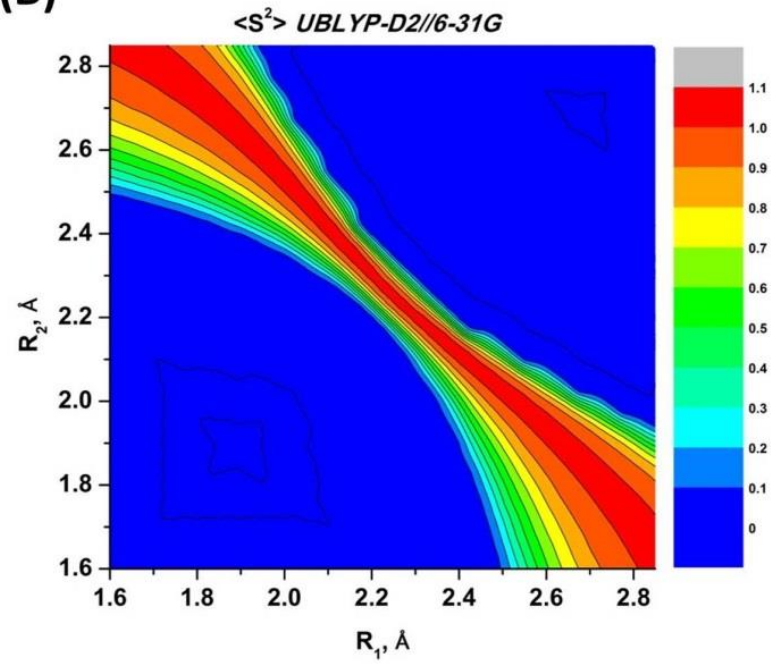

(D)

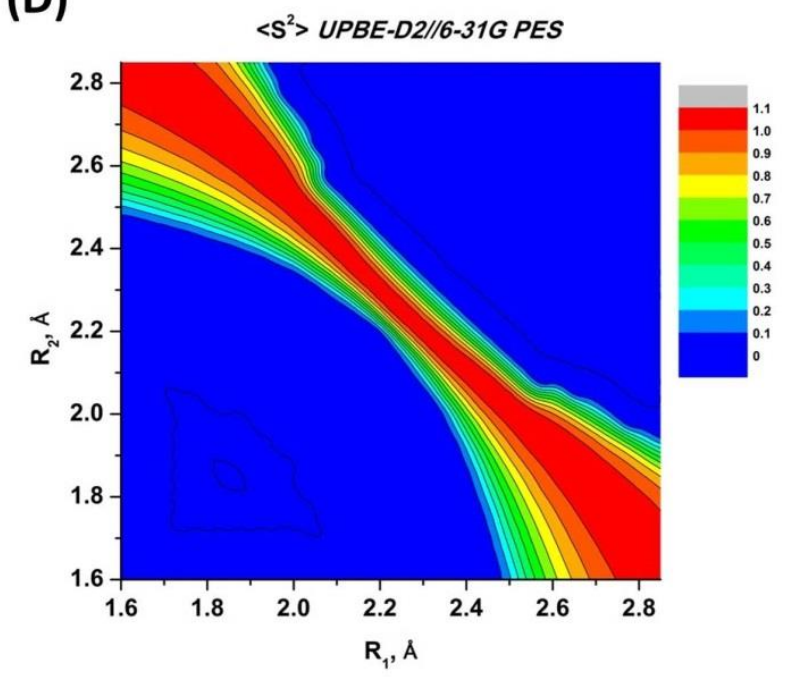

Figure $S_{3}<S^{2}>$ values corresponding to the potential energy surfaces in vacuum shown in Figure $S_{2}$. 
(A)

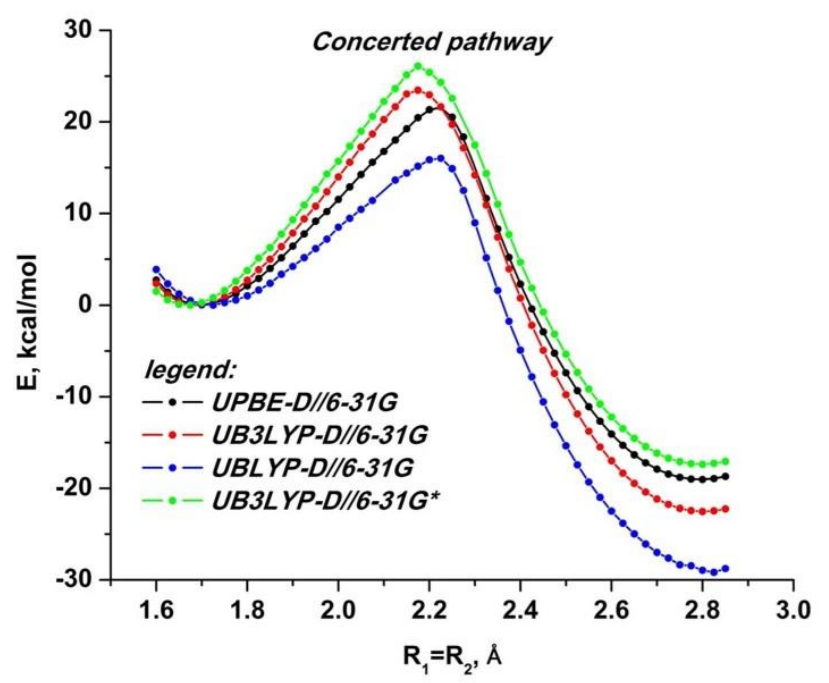

(B)

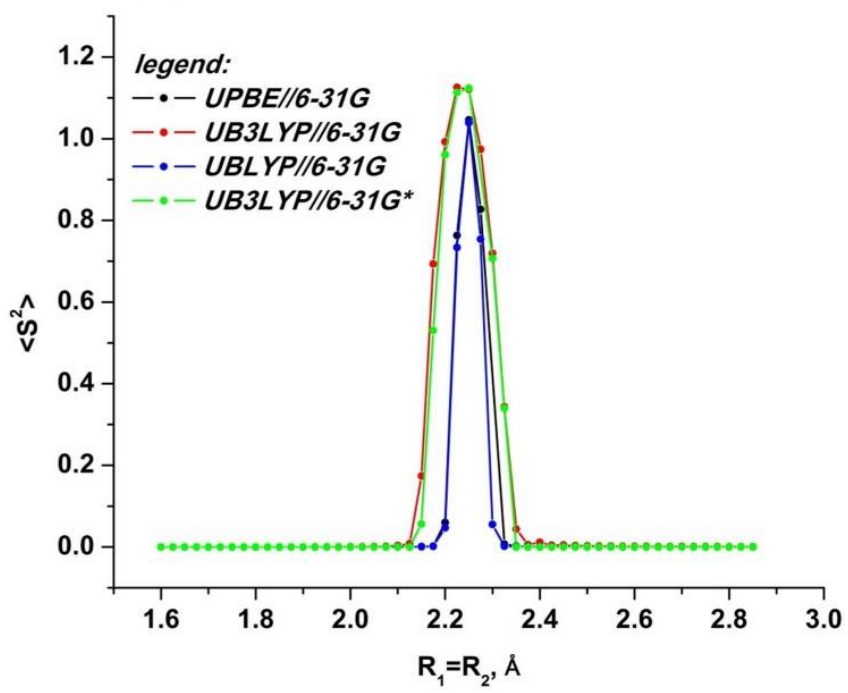

Figure $\mathrm{S}_{4}$ Concerted minimum energy pathways (A) and the corresponding expectation values of spin-square operator, $\left.<\mathrm{S}^{2}\right\rangle$, (B) computed for PI to BA reaction in vacuum from unrestricted DFT.

(A)

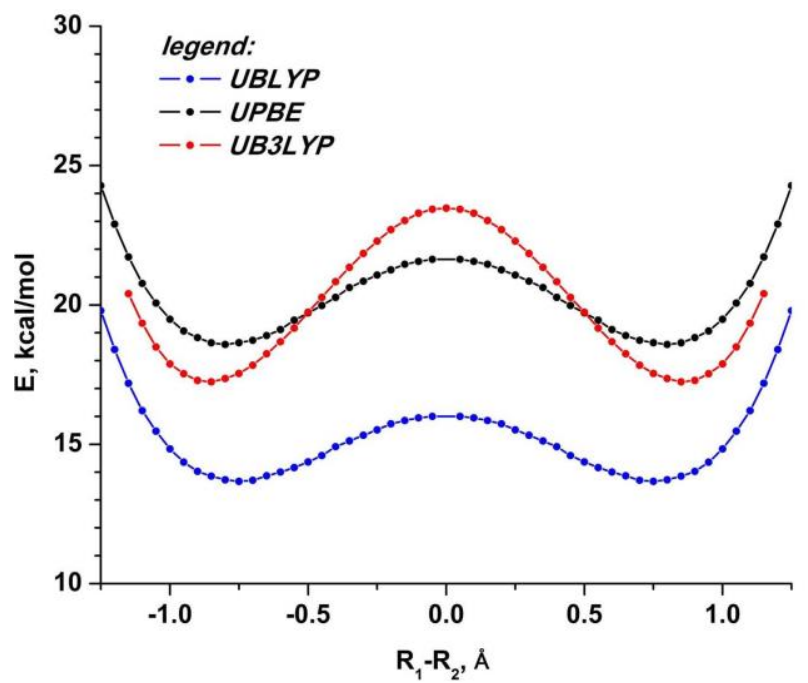

(B)

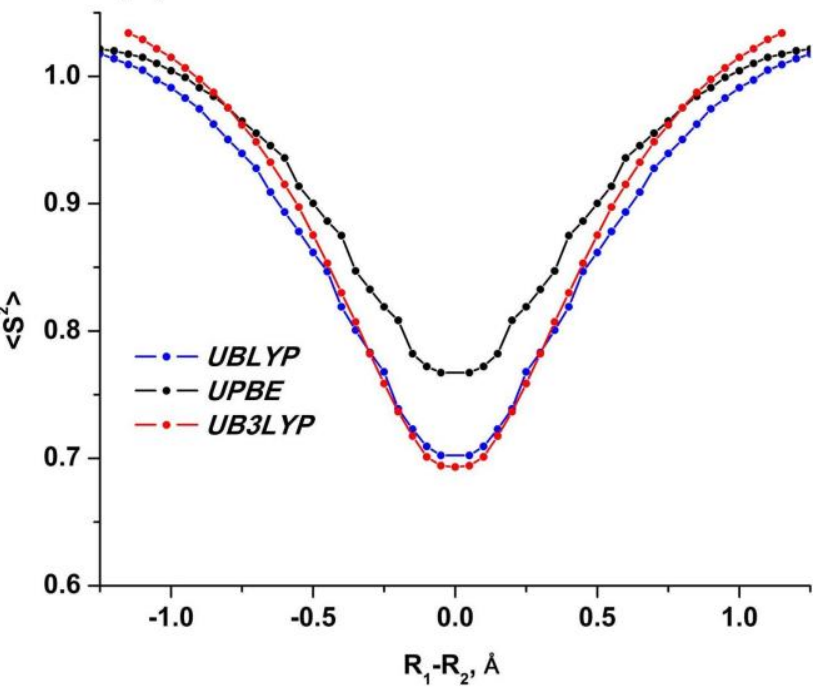

Figure $\mathrm{S}_{5}$ Projection of $2 \mathrm{D}$ energy surfaces at the stepwise TS regions (A) and the corresponding $\left\langle\mathrm{S}^{2}\right\rangle$ values (B) computed for PI-> BA reaction in vacuum from unrestricted DFT. 
(A)

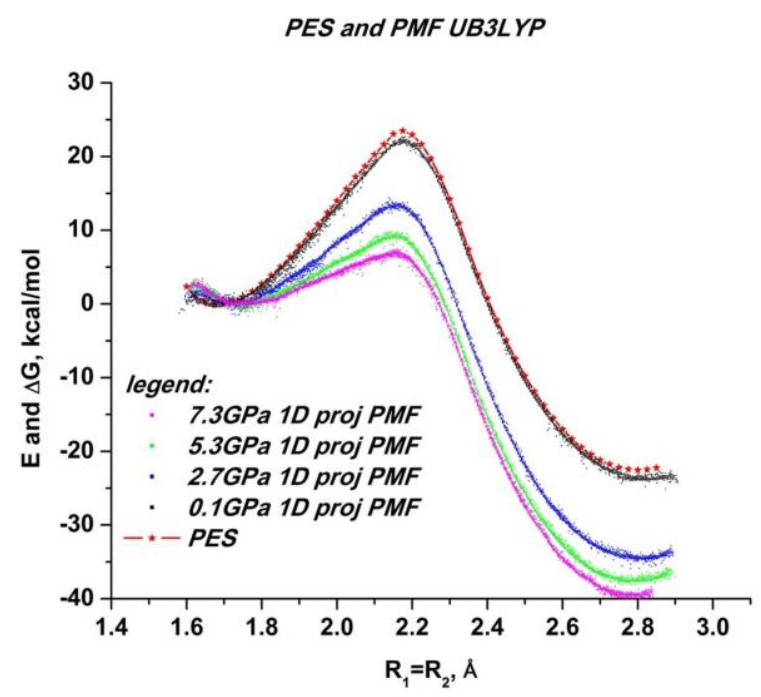

(B)

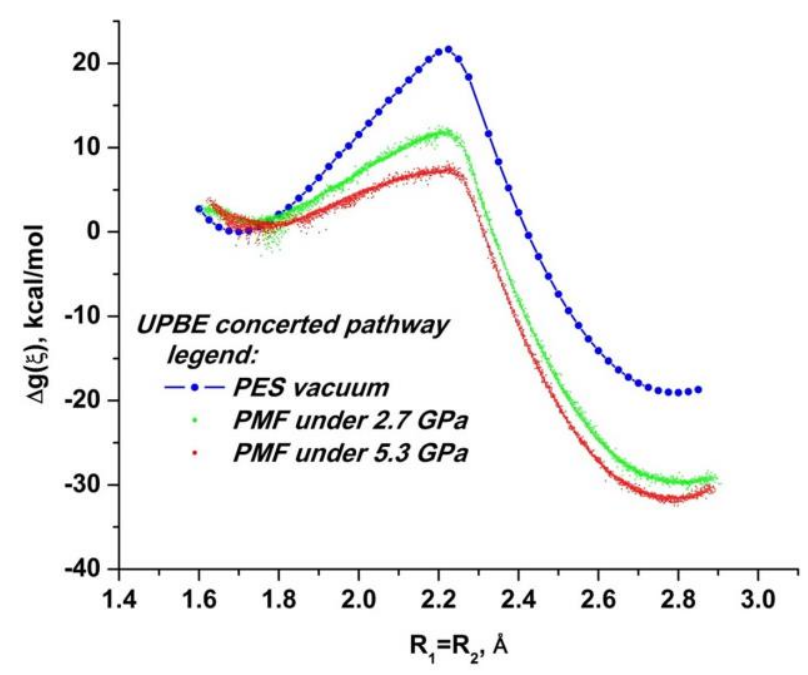

Figure S6 (A)Free energy paths at o.1 GPa; 2.7 GPa, 5.3 GPa, 7.3 GPa and the potential energy path in vacuum for the concerted mechanism computed using unrestricted, dispersion-corrected B3LYP//6-31G functional. (B) Free energy paths at 2.7 GPa and at 5.3 GPa and the potential energy path in vacuum for the concerted mechanism computed using unrestricted, dispersion-corrected PBE//6-31G functional.
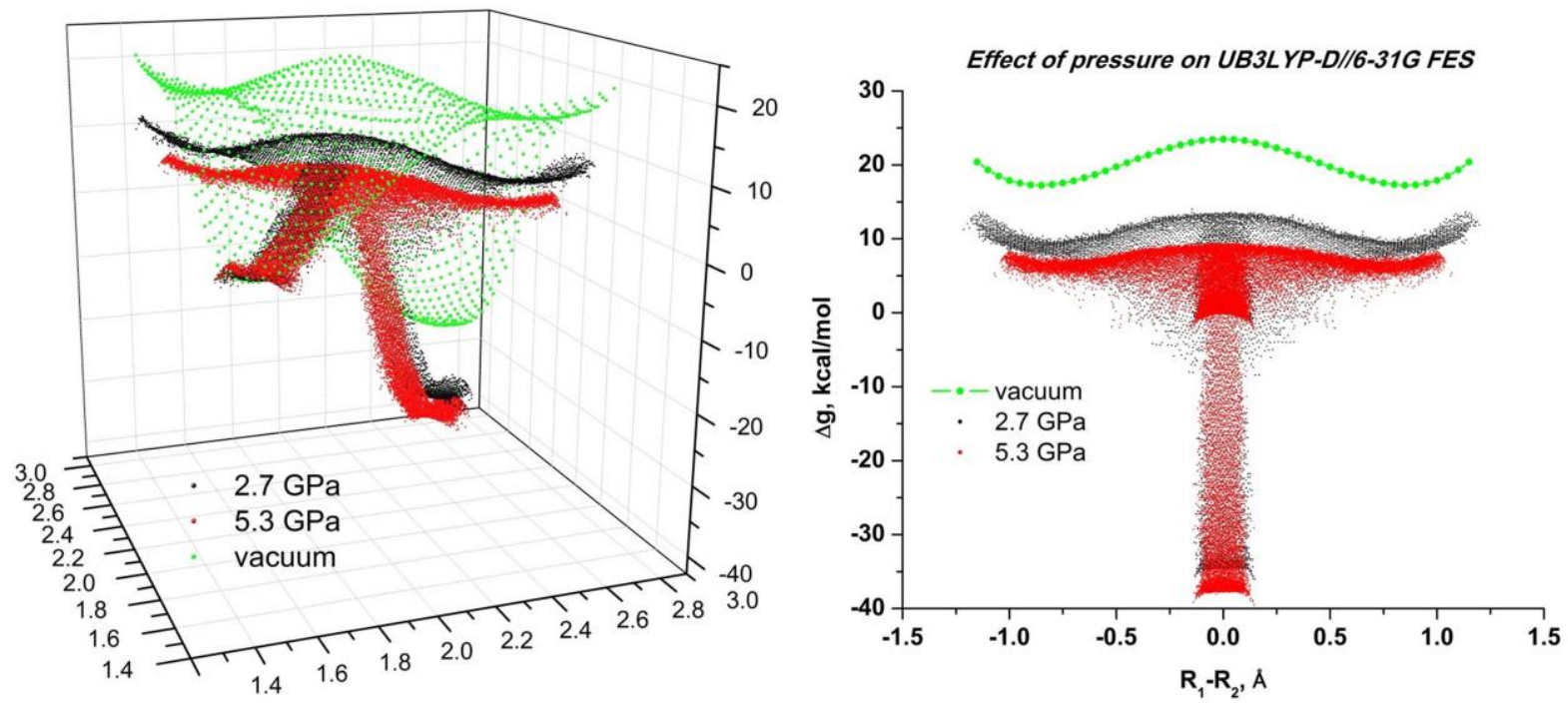

Figure $\mathrm{S}_{7}$ Effect of pressure on topology of the free energy surfaces. Given are potential energy map in vacuum (green) and free energy surfaces at 2.7 GPa (black) and at 5.3 GPa (red). 


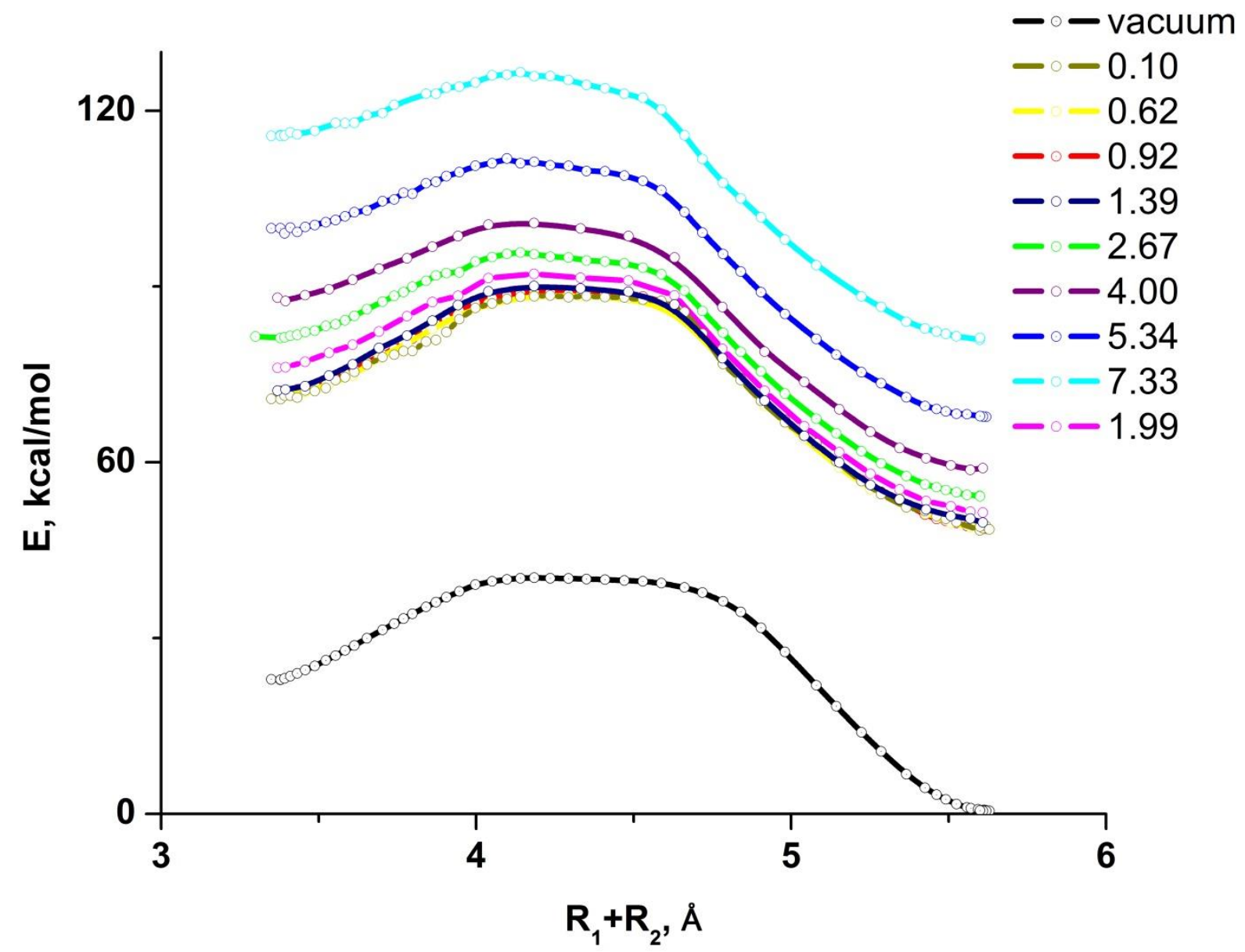

Figure S8 Increase of the average QM energy (over corresponding MD trajectories at 30oK) with pressure along the reaction path relative to the minimum energy path in vacuum (black line at the bottom). 


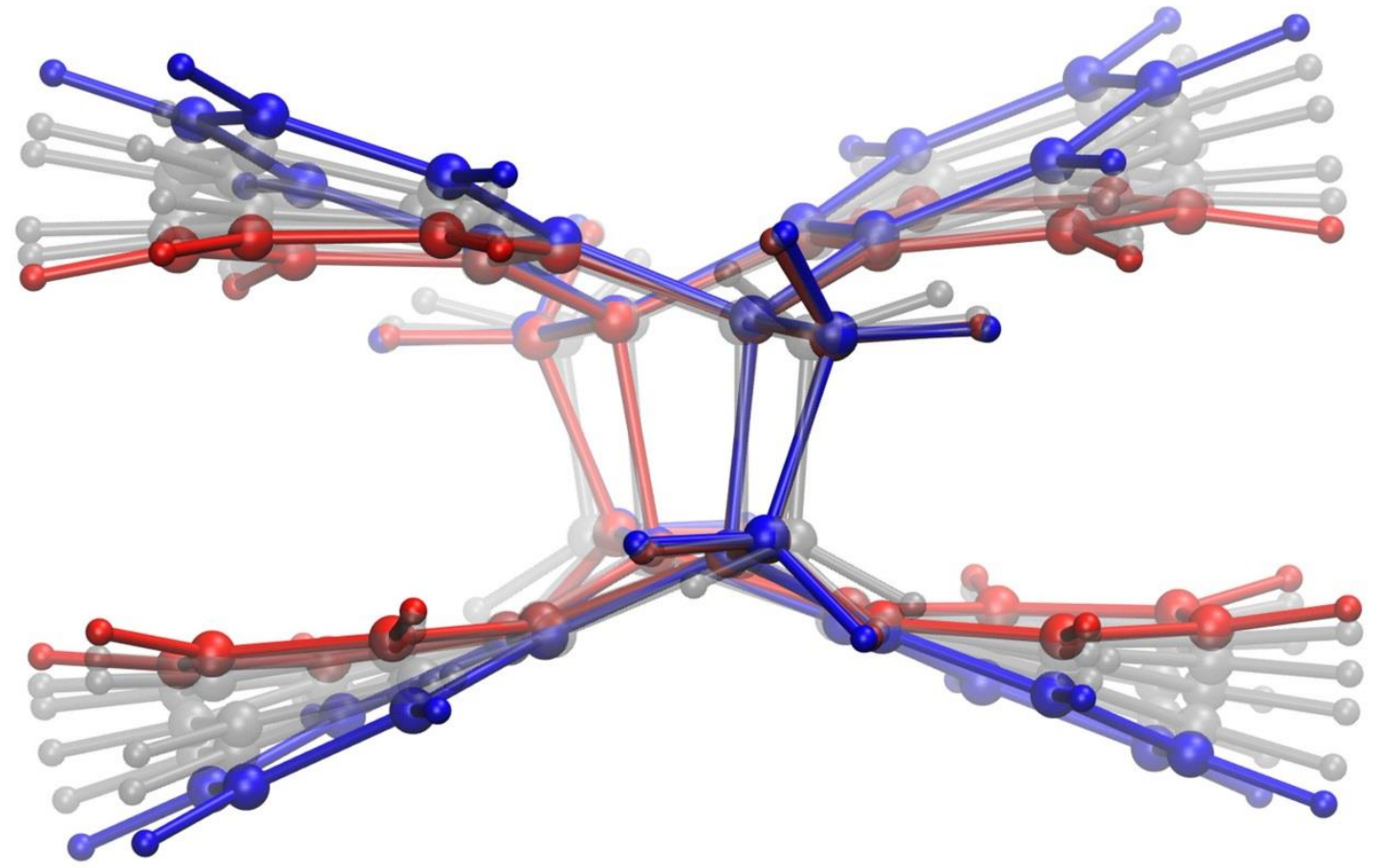

Figure $\mathrm{S}_{9}$ Change in averaged structure of the RS in response to the increased pressure relative to the minimum energy geometry in vacuum (blue ball and sticks). Red ball and sticks show the averaged structure from molecular dynamics at 7.33 GPa. Averaged structures at intermediate pressures are shown with grey balls and sticks. 


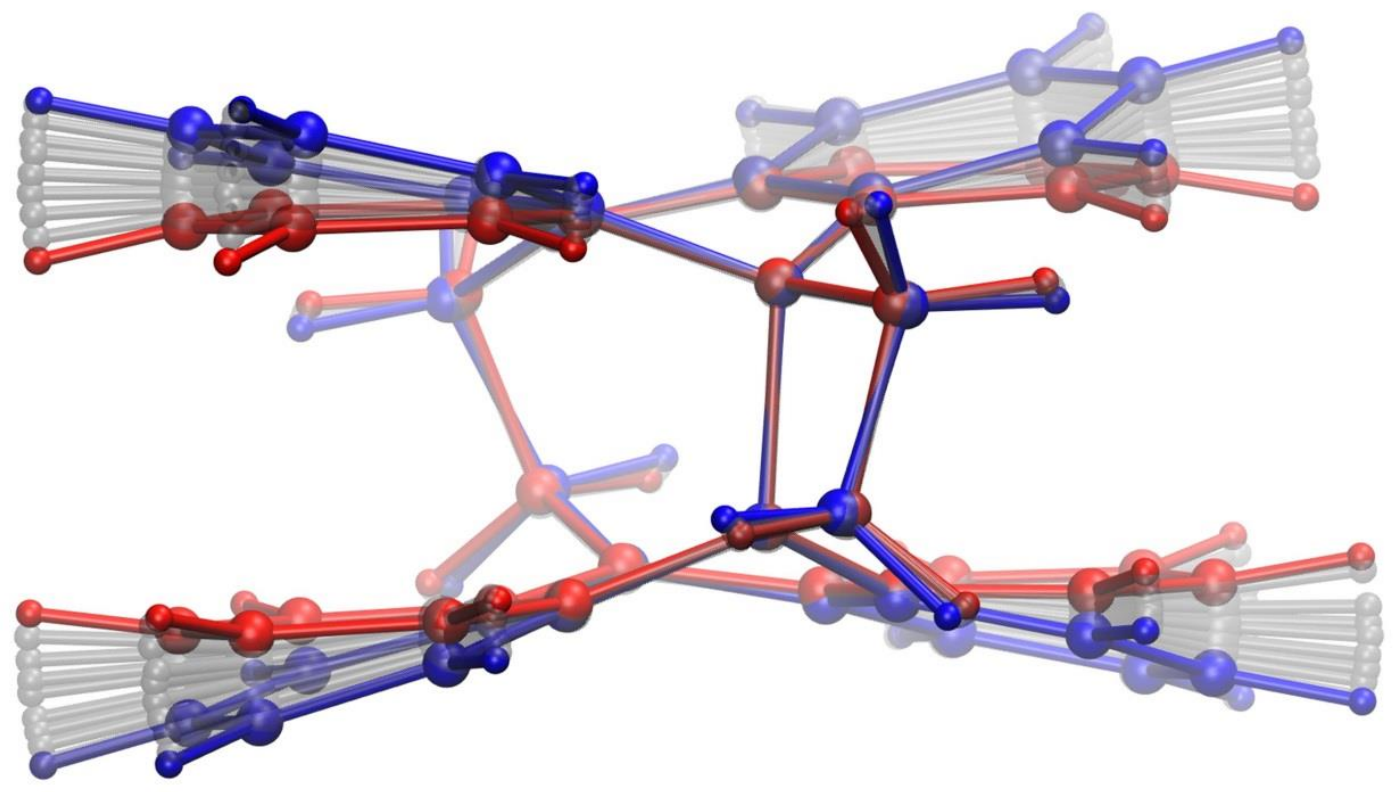

Figure Sio Change in averaged structure of the TS in response to the increased pressure relative to the minimum energy geometry in vacuum (blue ball and sticks). Red ball and sticks show the averaged structure from molecular dynamics at 7.33 GPa. Averaged structures at intermediate pressures are shown with grey balls and sticks. 


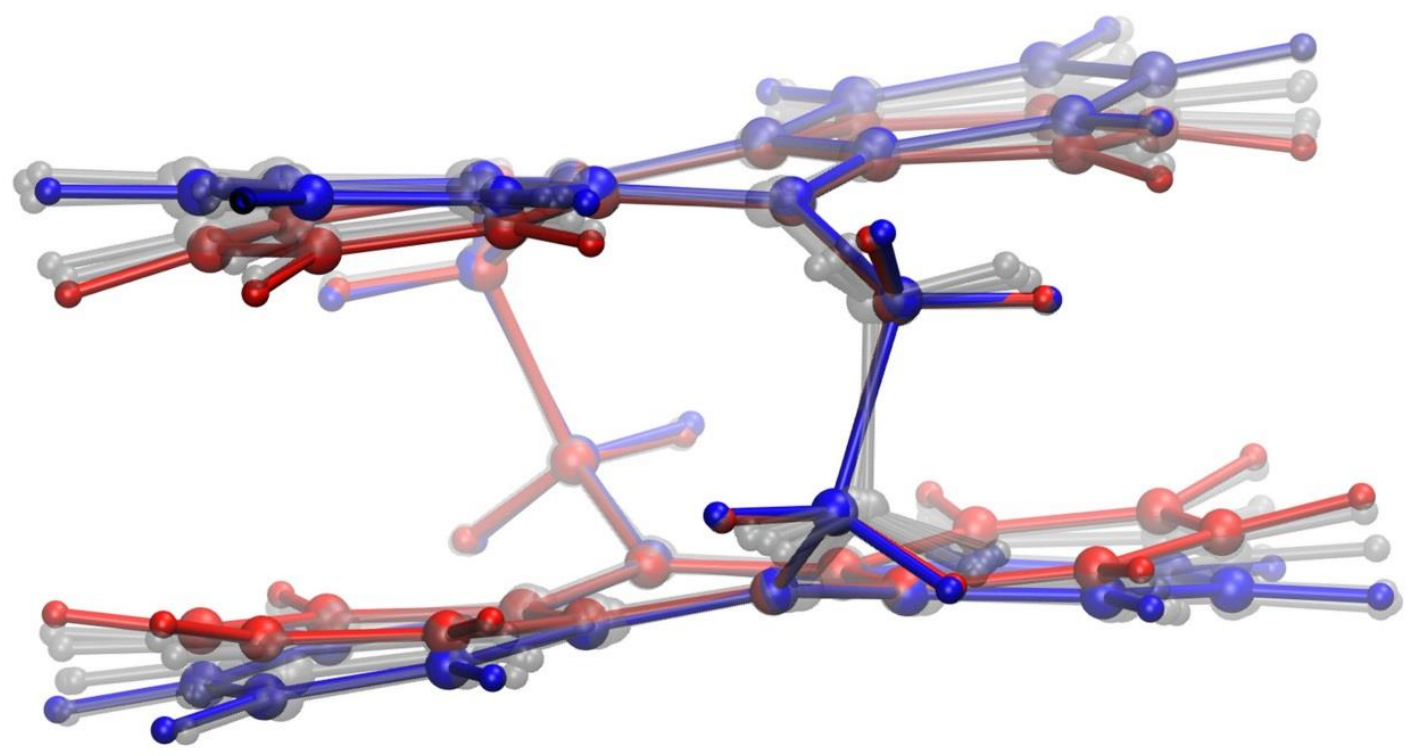

Figure S11 Change in averaged structure of the PS in response to the increased pressure relative to the minimum energy geometry in vacuum (blue ball and sticks). Red ball and sticks show the averaged structure from molecular dynamics at $7 \cdot 33 \mathrm{GPa}$. Averaged structures at intermediate pressures are shown with grey balls and sticks. 


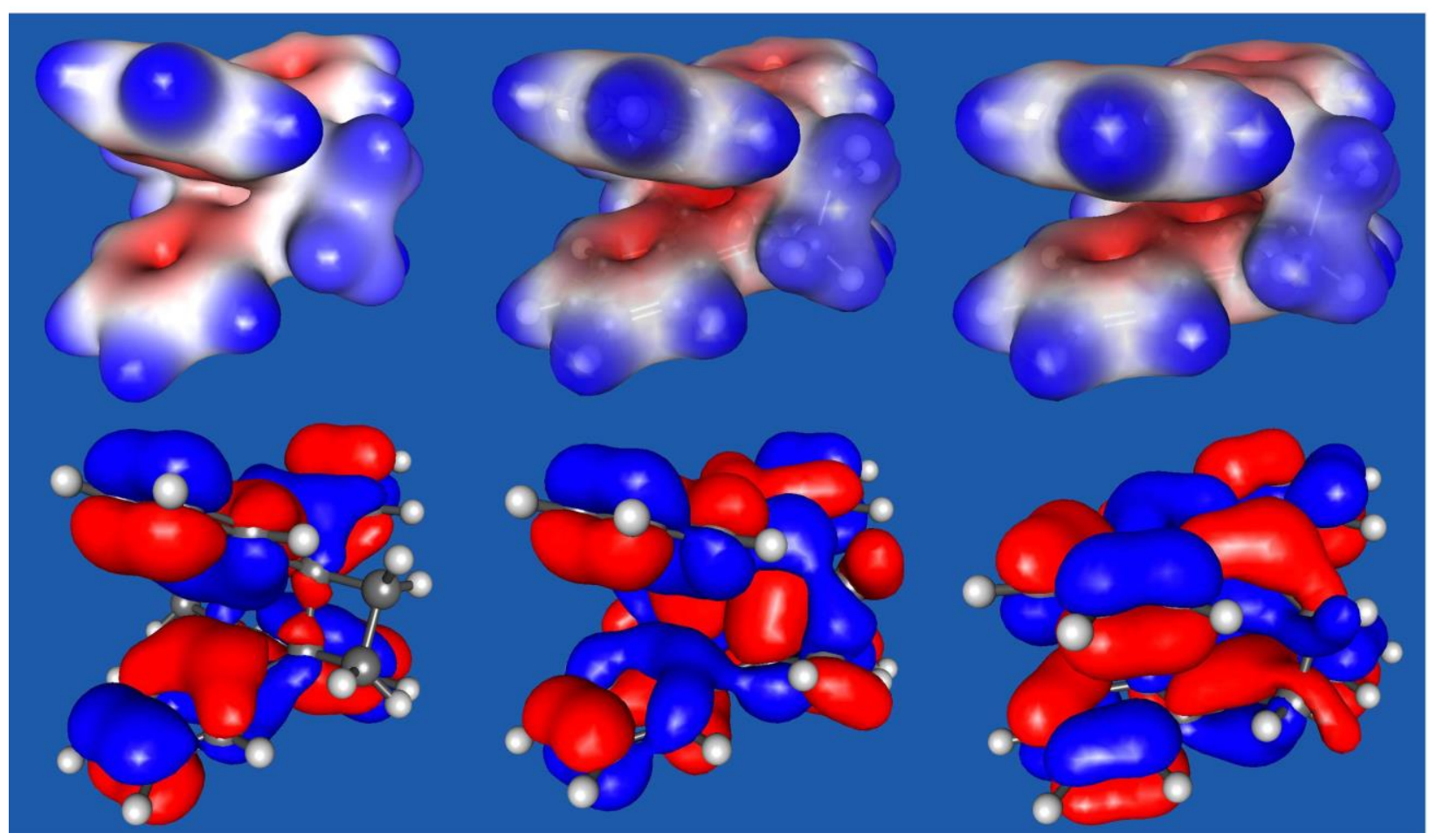

Figure S12 Top panel shows distribution of the charge density in RS, TS and PS. The bottom panel shows HOMO of RS, TS and PS. 


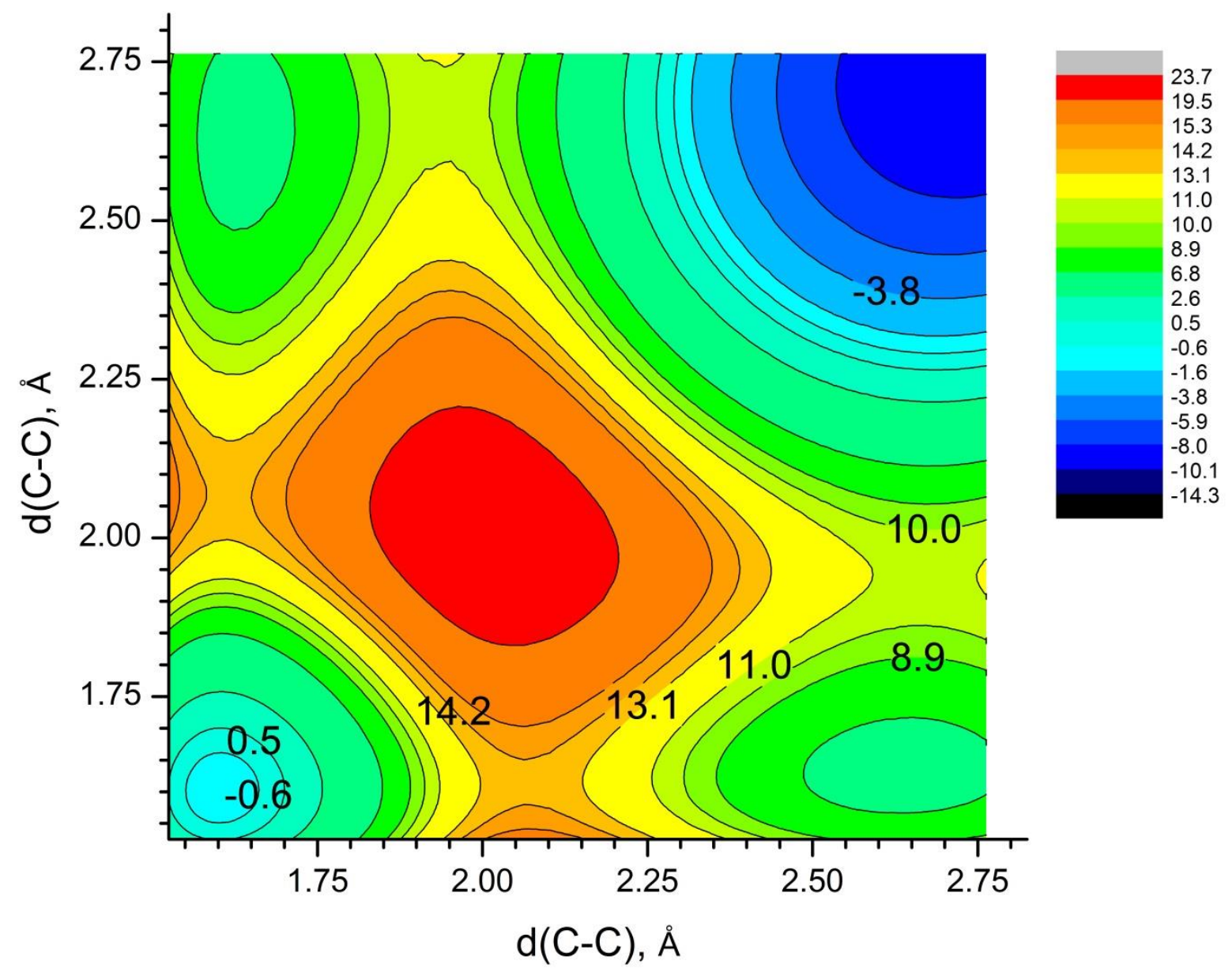

Figure $\mathrm{S}_{13} \mathrm{UPM}_{3}$ PES predicts a stable diradical intermediate. 

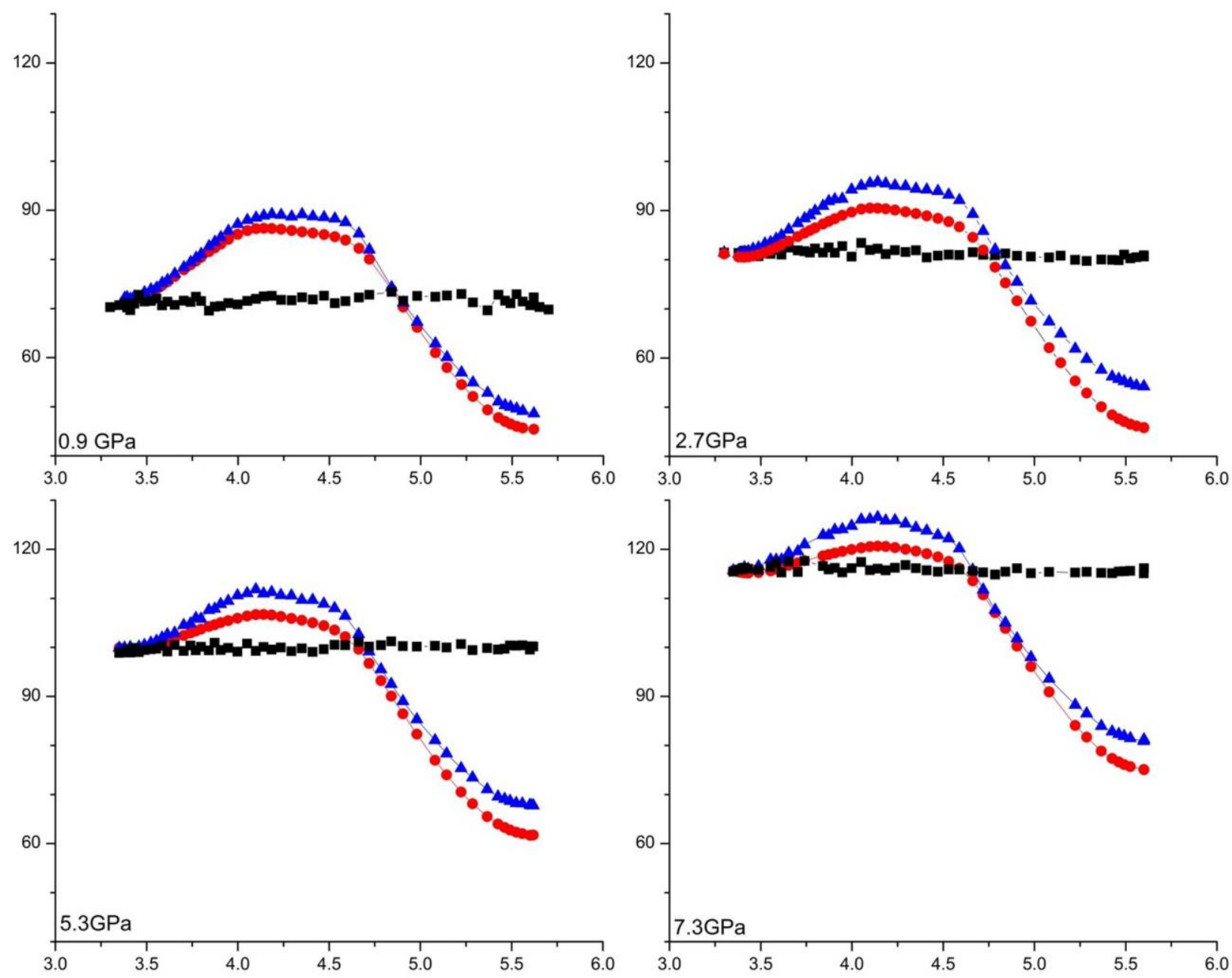

Figure $\mathrm{S}_{4} 4$ Gibbs free energy profiles (red circles) along the reaction path at different pressures and the corresponding entropic (black squares) and the internal energy (blue traingles) contributions. All energies are given relative to the minimum energy of the product state in vacuum. The deviation between the free energy and the potential energy increases with pressure for both the activation barrier and for the reaction energy change (o.9 GPa - top right panel), reaching maximum at $2.67 \mathrm{GPa}$ (top right panel). High pressures strongly affect the internal energy distribution by causing the RS destabilization (bottom left panel corresponds to $5.3 \mathrm{GPa}$ and bottom right panel to $7.3 \mathrm{GPa}$ ). 

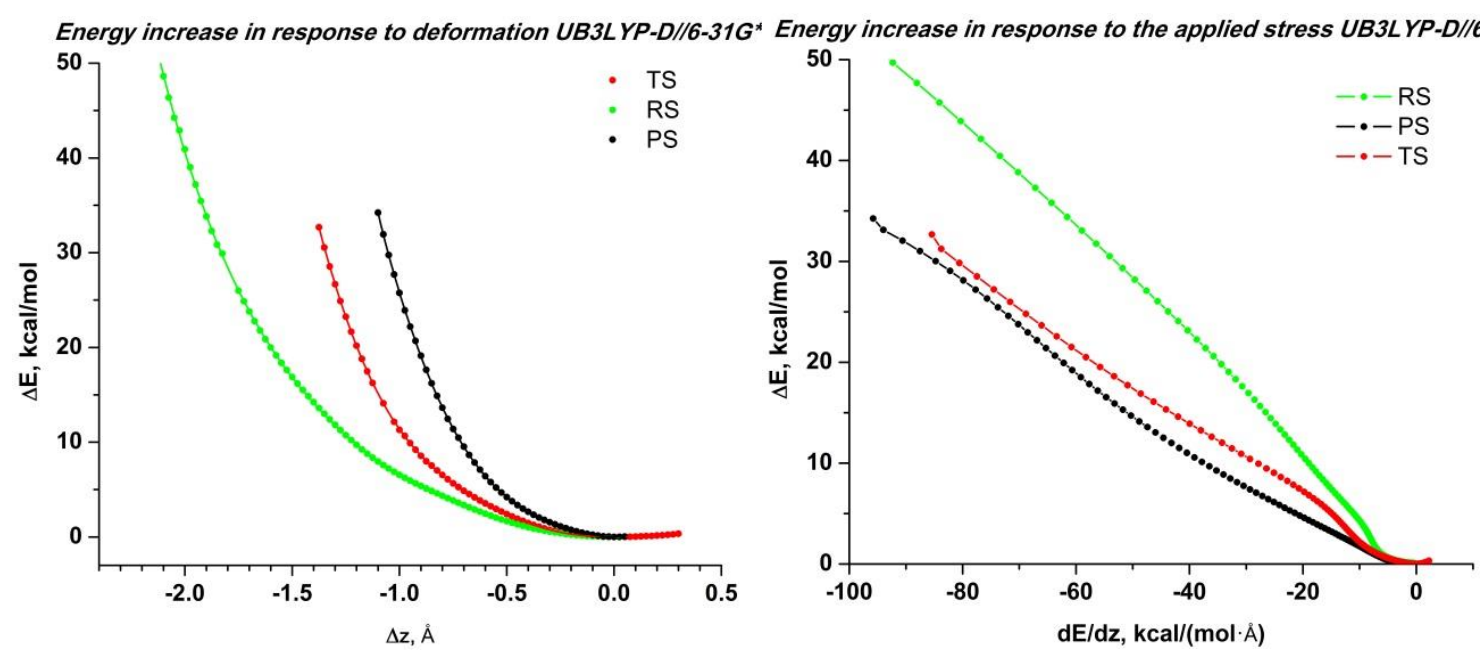

Figure S15 Left panel: UB3LYP-D2//6-31G* energies versus deformation along the z-coordinate (which is shown in Figure 1 of the main text) of the reactant state (RS, green), of the transition state on the concerted path (TS, red), and of the product state (PS, black). Right panel indicates by how much energies for these states increase in response to force applied along z-direction.

\section{References}

(1) Jezowski, S. R.; Zhu, L.; Wang, Y.; Rice, A. P.; Scott, G. W.; Bardeen, C. J.; Chronister, E. L. Pressure Catalyzed Bond Dissociation in an Anthracene Cyclophane Photodimer J. Am. Chem. Soc. 2012, $134,7459-7466$.

(2) Trzop, E.; Turowska-Tyrk, I. Monitoring Structural Transformations in Crystals. 12. Course of an Intramolecular [4 + 4] Photocycloaddition in a Crystalfor Part 11 See Turowska-Tyrk, Bakowicz \& Scheffer (2007) Acta Crystallographica Section B 2008, 64, 375-382.

(3) Jones Ii, G.; Reinhardt, T. E.; Bergmark, W. R. Photon Energy Storage in Organic Materials - the Case of Linked Anthracenes Solar Energy 1978, 20, 241-248.

(4) Mau, A. W. H. Solid State Reversible Reactions. Thermal Behaviour of the Photoisomer of Bi[Anthracene-9,10-Dimethylene] J. Chem. Soc., Faraday Trans. 1 1978, 74, 603-612.

(5) Slepetz, B.; Kertesz, M. Volume Change During Thermal [4 + 4] Cycloaddition of [2.2] $(9,10)$ Anthracenophane J. Am. Chem. Soc. 2013, 135, 13720-13727.

(6) Shao, Y.; Molnar, L. F.; Jung, Y.; Kussmann, J.; Ochsenfeld, C.; Brown, S. T.; Gilbert, A. T. B.; Slipchenko, L. V.; Levchenko, S. V.; O'Neill, D. P.et al. Advances in Methods and Algorithms in a Modern Quantum Chemistry Program Package Phys. Chem. Chem. Phys. 2006, 8, 3172-3191.

(7) Stewart, J. J. P.; Stewart Computational Chemistry: Colorado Springs, CO, USA, 2012.

(8) Pulay, P. Improved Scf Convergence Acceleration J. Comput. Chem. 1982, 3, 556-560.

(9) Hu, X.; Yang, W. Accelerating Self-Consistent Field Convergence with the Augmented Roothaan-Hall Energy Function J. Chem. Phys. 2010, 132, -.

(10) Grimme, S. Semiempirical Gga-Type Density Functional Constructed with a Long-Range Dispersion Correction J. Comput. Chem. 2006, 27, 1787-1799.

(11) Henkelman, G.; Jónsson, H. Improved Tangent Estimate in the Nudged Elastic Band Method for Finding Minimum Energy Paths and Saddle Points J. Chem. Phys. 2000, 113, 9978-9985.

(12) Ufimtsev, I. S.; Martinez, T. J. Quantum Chemistry on Graphical Processing Units. 3. Analytical Energy Gradients, Geometry Optimization, and First Principles Molecular Dynamics J. Chem. Theory Comput. 2009, 5, 2619-2628.

(13) Kästner, J.; Carr, J. M.; Keal, T. W.; Thiel, W.; Wander, A.; Sherwood, P. DI-Find: An OpenSource Geometry Optimizer for Atomistic Simulationst J. Phys. Chem. A 2009, 113, 11856-11865. 
(14) Plotnikov, N. V. Computing the Free Energy Barriers for Less by Sampling with a Coarse Reference Potential While Retaining Accuracy of the Target Fine Model J. Chem. Theory Comput. 2014.

(15) Grossfield, A.; WHAM 2.0.8 ed.; University of Rochester: Rochester, NY, 2013.

(16) Grünwald, M.; Dellago, C. Ideal Gas Pressure Bath: A Method for Applying Hydrostatic Pressure in the Computer Simulation of Nanoparticles Mol. Phys. 2006, 104, 3709-3715.

(17) Grünwald, M.; Dellago, C.; Geissler, P. L. An Efficient Transition Path Sampling Algorithm for Nanoparticles under Pressure J. Chem. Phys. 2007, 127, - .

(18) Saucier, R. Computer Generation of Statistical Distributions, Army Research Laboratory, 2000.

(19) Park, S. K.; Miller, K. W. Random Number Generators: Good Ones Are Hard to Find Commun. ACM 1988, 31, 1192-1201.

(20) Landau, L. D.; Lifshitz, E. M. Statistical Physics; Pergamon Press: Oxford, 1969; Vol. 5.

(21) Ribas-Arino, J.; Marx, D. Covalent Mechanochemistry: Theoretical Concepts and Computational Tools with Applications to Molecular Nanomechanics Chem. Rev. 2012, 112, 5412-5487.

(22) Beyer, M. K. The Mechanical Strength of a Covalent Bond Calculated by Density Functional Theory J. Chem. Phys. 2000, 112, 7307-7312.

(23) Hunter, C. A.; Sanders, J. K. M. The Nature Of .Pi.-.Pi. Interactions J. Am. Chem. Soc. $1990,112,5525-5534$. 


\section{XYZ coordinates for RS, TS, PS RS UB3LYP-D2//6-31G from potential energy scan}

56

$<\mathrm{S}_{2}>=0.0001 \mathrm{ESCF}=-1233.6432851820 \quad \mathrm{R} 1=1.675 \mathrm{R} 2=1.675$

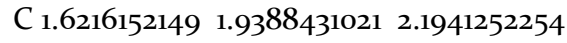

C $0.8320079158 \quad 1.4427186910 \quad 1.1576380285$

$\begin{array}{lllll}C-0.5765008394 & 1.3876097083 & 1.2931458032\end{array}$

C - $1.1569577168 \quad 1.8544924952 \quad 2.4754982344$

C - $0.3615340812 \quad 2.3591645290 \quad 3.5154179198$

C $1.0268470722 \quad 2.4005785816 \quad 3.3774564701$

$\begin{array}{llll}C-1.3697783459 & 0.8297070115 & 0.1139516191\end{array}$

C - $0.83201594991 .4427133339-1.1576383807$

C $0.5764931501 \quad 1.3876134602-1.2931458748$

$\begin{array}{llll}C & 1.3697738579 & 0.8297153320 & -0.1139518689\end{array}$

C $1.1569475146 \quad 1.8545009266-2.4754976837$

$\begin{array}{llll}C & 0.3615209882 & 2.3591681219 & -3.5154174842\end{array}$

C - $1.0268605331 \quad 2.4005724238$-3.3774567338

C - $1.6216261176 \quad 1.9388326859-2.1941258655$

$\begin{array}{llll}C & 2.9096509009 & 0.7197961838 & -0.2830054497\end{array}$

$\begin{array}{lllll}C & 2.9096545433 & -0.7197796189 & 0.2830053101\end{array}$

$\begin{array}{lllll}C & 1.3697785885 & -0.8297076927 & 0.1139521442\end{array}$

C $0.5765009886-1.3876093874 \quad 1.2931455608$

C - $0.8320077990 \quad-1.4427185916 \quad 1.1576378308$

C -1.3697741021 -0.8297160156 -0.1139523766

C - $0.5764932996-1.3876131451-1.2931456183$

C $0.8320158334-1.4427132373-1.1576381682$

$\begin{array}{llll}C & 1.1569577178 & -1.8544921963 & 2.4754981253\end{array}$

C $0.3615340244-2.3591644250 \quad 3.5154181053$

C - $1.0268474083-2.40057850253 .3774565343$

C - $1.6216152127-1.93884314692 .1941253529$

C $-1.1569475140 \quad-1.8545006263-2.4754975664$

C -0.3615209311 -2.3591680099 -3.5154176644

C $1.0268608691-2.4005723366-3.3774567919$

C $1.6216261143-1.9388327265-2.1941259838$

C -2.9096505703 $-0.7197965500 \quad-0.2830056344$

$\begin{array}{llll}C-2.9096548746 & 0.7197792552 & 0.2830051176\end{array}$

H $2.7010963030-1.9622302532-2.0916630151$

H $1.6467257970-2.7874145148-4.1806786940$

H - $0.8320759064 \quad-2.7157804578-4.4267545949$

$\mathrm{H}-2.2318328536-1.8352028403-2.6075292905$

H $3.5148177603-1.4623637452-0.2439981588$

$\begin{array}{llll}\text { H } 3.2005538690 & -0.7253509123 & 1.3344844526\end{array}$

$\mathrm{H}-2.7010851923-1.96224774092 .0916618327$

H -1.6467100821 $-2.7874248210 \quad 4.1806781819$

H o.8320909817 -2.7157730087 4.4267555226

H $2.2318428183-1.83518680522 .6075306099$

$\mathrm{H}-3.2005498371-0.7253695483-1.3344847881$

$\begin{array}{llll}\mathrm{H}-3.5148097370 & -1.4623839777 & 0.2439978364\end{array}$

H -2.7010963133 $1.9622301452 \quad-2.0916630881$

H $-1.6467256857 \quad 2.7874145156 \quad-4.1806785327$

H o.8320757736 2.7157805745 -4.4267544543

H $2.2318329420 \quad 1.8352030659$-2.6075289238

$\mathrm{H}-3.5148176784 \quad 1.4623639320-0.2439978766$

$\begin{array}{llll}\mathrm{H}-3.2005533565 & 0.7253506920 & 1.3344848589\end{array}$

H 2.7010852019 1.96224762852 .0916619018

H $1.6467099707 \quad 2.7874248109$ 4.1806780171

H -o.83209o8486 2.7157731136 4.4267553787

H -2.2318429058 1.83518702672 .6075302393 
$\begin{array}{llll}\text { H } 3.2005493323 & 0.7253693217 & -1.3344851998\end{array}$

$\mathrm{H}_{3.5148096485} 1.4623841654 \quad 0.2439975484$

TS along the concerted pathway, UB3LYP-D2//6-31G from potential energy scan

56

$<\mathrm{S}_{2}>=0.6333 \mathrm{ESCF}=-\mathbf{1 2 3 3} .6054703879 \mathrm{R} \mathbf{1}=\mathbf{2 . 1 7 5} \mathrm{R} 2=\mathbf{2} .175$

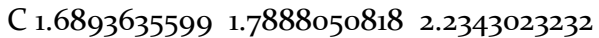

C $0.8620501621 \quad 1.48412069451 .1440921210$

C - $-0.5578518586 \quad 1.46061236621 .3187561626$

C - $-1.08692356331 .7635300352 \quad 2.5830858543$

C - -0.24928331212 .09366608673 .6570379540$

C 1.13897555962 .10249522713 .4844743836

C $-1.3767658412 \quad 1.0751764650 \quad 0.1632329951$

C -0.8620591482 $1.4841106467-1.1440956070$

C $0.5578401044 \quad 1.4606101846-1.3187595308$

C $1.3767586331 \quad 1.0751835931-0.1632307959$

$\begin{array}{llll}C & 1.0869112878 & 1.7635340810 & -2.5830874464\end{array}$

C $0.24926928292 .0936664355-3.6570382718$

C - $1.13898831042 .1024888510-3.4844739063$

C - $-1.6893744448 \quad 1.7887950165 \quad-2.2343026766$

$\begin{array}{llll}C & 2.8525817870 & 0.6977212011 & -0.3318650249\end{array}$

$\begin{array}{lllll}C & 2.8525860470 & -0.6977047671 & 0.3318657036\end{array}$

$\begin{array}{llll}C & 1.3767658345 & -1.0751760224 & 0.1632328640\end{array}$

$\begin{array}{llll}C & 0.5578517289 & -1.4606118937 & 1.3187560379\end{array}$

$\begin{array}{llll}C & -0.8620500582 & -1.4841203093 & 1.1440920284\end{array}$

C - $1.3767585980-1.0751831281-0.1632308211$

C $-0.5578399664-1.4606096566-1.3187595763$

C $0.8620590511-1.4841102540-1.1440956753$

$\begin{array}{llll}C & 1.0869237538 & -1.7635299891 & 2.5830859509\end{array}$

C 0.2492832089 -2.0936661273 3.6570377367

C - $1.1389753132-2.1024956208 \quad 3.4844741465$

C - $1.6893637068-1.7888054346 \quad 2.2343023220$

C -1.086911488o $-1.7635340316-2.5830876899$

C - $0.2492692022-2.0936666278-3.6570381630$

C $1.1389880441-2.1024894553-3.4844737968$

C $1.6893745844-1.7887954715-2.2343028298$

C - $2.8525814452-0.6977209099-0.3318651964$

$\begin{array}{lllll}C-2.8525863600 & 0.6977050017 & 0.3318656644\end{array}$

H $2.7665389383-1.7905564802-2.1025552873$

H $1.7923178254-2.3504953695-4.3156550519$

$\mathrm{H}-0.6829308805-2.3365758020-4.6221947805$

$\mathrm{H}-2.1601836391 \quad-1.7632355202-2.7345827450$

H $3.5599037717-1.4130636709-0.1069008852$

$\begin{array}{lllll}\text { H } 3.0935523544 & -0.5994917455 & 1.3913091088\end{array}$

$\mathrm{H}-2.7665282817$-1.7905686629 2.1025562128

$\mathrm{H}-1.7923035428 \quad-2.35049965544 .3156572291$

H o.6829461762 -2.3365686629 4.6221957756

H $2.1601953919-1.7632232101 \quad 2.7345830562$

$\mathrm{H}-3.0935470887-0.5995101501-1.3913089501$

$\begin{array}{llll}\mathrm{H}-3.5598946602 & -1.4130845703 & 0.1069012303\end{array}$

$\mathrm{H}-2.76653895641 .7905558777 \quad-2.1025552468$

$\begin{array}{llll}\mathrm{H}-1.7923179178 & 2.3504940765 & -4.3156551958\end{array}$

Н o.6829307446 2.3365752502 -4.6221948436

H 2.1601836088 1.7632358990 -2.7345825942

H -3.559903886o $1.4130643114-0.1069008620$

$\begin{array}{llll}\mathrm{H}-3.0935524262 & 0.5994921121 & 1.3913092735\end{array}$

H 2.7665282815 1.79056816922 .1025563556

H 1.79230359912 .35049870334 .3156574840

$\mathrm{H}-0.6829460785 \quad 2.33656833254 .6221959180$ 
$\begin{array}{llll}\mathrm{H}-2.1601953843 & 1.7632235272 & 2.7345830481\end{array}$

$\begin{array}{llll}\text { H } 3.0935472482 & 0.5995107868 & -1.3913089939\end{array}$

H 3.55989478911 .41308518540 .1069015042

TS along the sequential (minimum energy) pathway, UB3LYP-D2//6-31G from potential energy scan

56

$<\mathrm{S}_{2}>=0.7629 \mathrm{ESCF}=-1233.6150050638 \mathrm{R} 1=2.475 \mathrm{R}_{2}=1.725$

C $1.68807811111 .8152514243 \quad 2.2116093838$

C 0.87038351721 .48762067061 .1117862664

C $-0.5400849503 \quad 1.3858248711 \quad 1.2875790163$

C - $-1.0853267309 \quad 1.7067646575 \quad 2.5313060623$

C - $0.2670643610 \quad 2.08534937123 .6080216364$

C $1.12224682642 .1174551453 \quad 3.4527466854$

$\begin{array}{llll}C-1.3422489377 & 0.8549990771 & 0.1134749295\end{array}$

C - $0.8494691897 \quad 1.4594192581-1.1810717489$

C $0.57049514431 .5136879973-1.3456275970$

C $1.41368752861 .2206522599-0.2035273401$

C $1.09312704611 .8471242979-2.6124767616$

C $0.2468243532 \quad 2.1659555814-3.6771082575$

C -1.1406038566 2.1455543441 -3.4981778084

C $-1.67969770661 .7797349238 \quad-2.2535270790$

C $2.83447300640 .6946033356-0.3546623477$

C $2.8344768940-0.6945890160 \quad 0.3546646893$

C $1.4136940841-1.2206441606 \quad 0.2035313706$

$\begin{array}{llll}C & 0.5705073545 & -1.5136873542 & 1.3456251623\end{array}$

C $-0.8494605026-1.45942808531 .1810693450$

C - $1.3422442382-0.8550063246-0.1134755637$

C - $0.5400765893-1.3858234902-1.2875812691$

C $0.8703899628-1.4876100295-1.1117878839$

C $1.0931398332-1.84711710722 .6124783721$

C $0.2468382744-2.1659552373 \quad 3.6771079615$

C - $1.1405904380 \quad-2.14556348793 .4981766110$

C - $-1.6796865028 \quad-1.7797470818 \quad 2.2535252995$

C - $1.0853166331-1.7067693364-2.5313077375$

C - $0.2670525096-2.0853494467$-3.6080222038

$C_{1.1222576378}-2.1174468224-3.4527461786$

C $1.6880861607-1.8152397030-2.2116076664$

C - $2.8775532068-0.7120539126-0.3003115618$

$\begin{array}{lllll}C-2.8775569639 & 0.7120380457 & 0.3003108165\end{array}$

H $2.7644399468-1.8576633032-2.0800284671$

H 1.7603453679 -2.3826391847 -4.2901437442

$\mathrm{H}-0.7175269891-2.3322250912-4.5637645850$

H -2.1554511638 -1.6616154731 -2.6897612818

H $3.5978355067-1.3644899790-0.0655946597$

H $3.0618097191-0.55739924951 .4143720442$

$\begin{array}{llll}\mathrm{H}-2.7565763297 & -1.7227190641 & 2.1377922436\end{array}$

H -1.8024147861 -2.3929970747 4.3221634886

H o.669600o698 -2.4302316892 4.6414133284

$\begin{array}{llll}\text { H } 2.1676320574 & -1.8812958751 & 2.7546091873\end{array}$

$\begin{array}{llll}\mathrm{H}-3.1497985080 & -0.6832718542 & -1.3563702129\end{array}$

$\begin{array}{llll}\mathrm{H}-3.4971695340 & -1.4661565854 & 0.1938838084\end{array}$

$\mathrm{H}-2.7565873388 \quad 1.7227032772-2.1377935309$

$\mathrm{H}-1.8024297593 \quad 2.3929828678-4.3221652011$

H o.6695847305 2.4302315473 -4.6414143711

H 2.16761907251 .8813078759 -2.7546067393

H -3.4971777877 1.4661372999 -0.1938839408

$\begin{array}{llll}\mathrm{H}-3.1498018367 & 0.6832541526 & 1.3563694969\end{array}$

H 2.76443185361 .85768188462 .0800303783 
H 1.76033180162 .38265325694 .2901446944

$\mathrm{H}-0.7175407415 \quad 2.33222164614 .5637638652$

H -2.1554606o61 1.66160132102 .6897612083

$\begin{array}{llll}\text { H } 3.0618046649 & 0.5574162806 & -1.4143701027\end{array}$

$\begin{array}{llll}\text { H } 3.5978281731 & 1.3645083482 & 0.0655964900\end{array}$

\section{TS from NEB with 1 negative frequency}

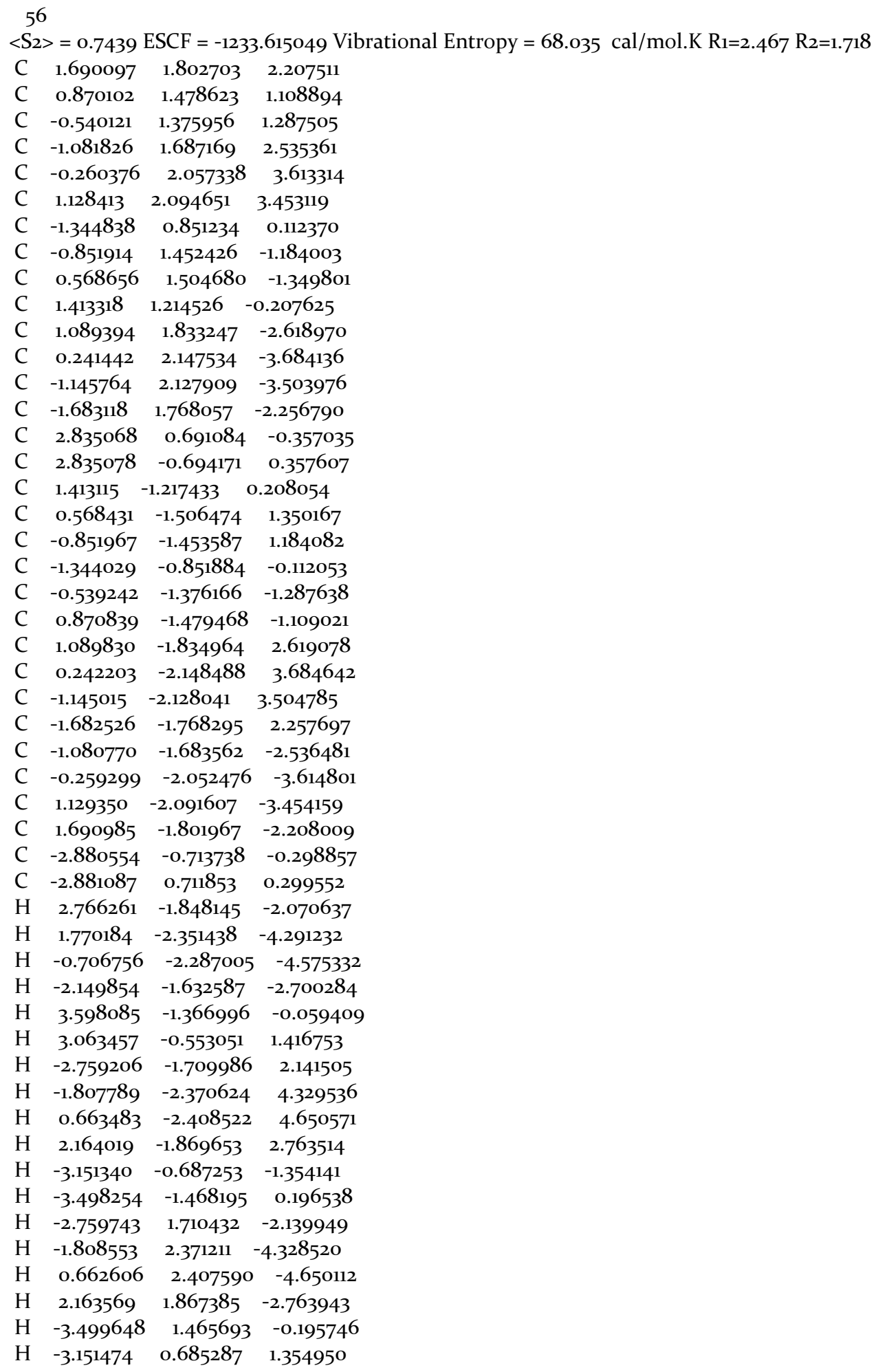

Plotnikov and Martinez-Page S26 


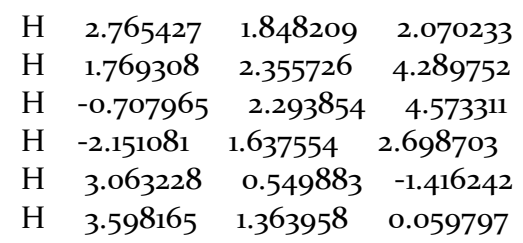

\section{PS, UB3LYP-D2//6-31G from potential energy scan}

56

$<\mathrm{S}_{2}>=0.0009 \mathrm{ESCF}=-1233.6792186224 \mathrm{R} 1=2.800 \mathrm{R} 2=2.800$

$\begin{array}{llll}C & 1.7225572550 & 1.7094793240 & 2.2544614532\end{array}$

C $0.8772644157 \quad 1.5846155578 \quad 1.1061140841$

C - $-0.5615350416 \quad 1.5653773991 \quad 1.3024670116$

C - $-1.0542822238 \quad 1.6768587797 \quad 2.6434655444$

C - $0.2067466935 \quad 1.8312087396 \quad 3.7149421856$

$\begin{array}{llll}C & 1.2030626519 & 1.8507699734 & 3.5187319447\end{array}$

$\begin{array}{lllll}C-1.4152103859 & 1.3875419648 & 0.1863217253\end{array}$

C - $0.8772722990 \quad 1.5846101943-1.1061144297$

C $0.56152818691 .5653802368-1.3024660423$

$\begin{array}{llll}C & 1.4152020869 & 1.3875502885 & -0.1863215935\end{array}$

$\begin{array}{llll}C & 1.0542735540 & 1.6768649625 & -2.6434646727\end{array}$

C $0.20673788351 .8312095734-3.7149419390$

C - $1.2030717404 \quad 1.8507617811-3.5187331997$

C - $1.7225668420 \quad 1.7094675059 \quad-2.2544638980$

$\begin{array}{llll}C & 2.7764889952 & 0.7277312525 & -0.3246149454\end{array}$

$\begin{array}{llll}C & 2.7764926490 & -0.7277148532 & 0.3246149502\end{array}$

$\begin{array}{llll}C & 1.4152091905 & -1.3875419663 & 0.1863217140\end{array}$

C $0.5615367822-1.5653769057 \quad 1.3024664031$

C -0.8772630505 -1.584616006o 1.1061145415

C - $1.4152032778-1.3875502879-0.1863215978$

C $-0.5615264491-1.5653807432-1.3024666479$

C $0.8772736578-1.5846097621-1.1061139764$

$\begin{array}{llll}C & 1.0542826776 & -1.6768588012 & 2.6434638921\end{array}$

C $0.2067483889-1.8312085763 \quad 3.7149415826$

C -1.2030613482 $-1.85077001453 .518733055^{2}$

$\begin{array}{llll}C-1.7225572852 & -1.7094792955 & \mathbf{2 . 2 5 4 4 6 3 2 1 6 1}\end{array}$

C - $1.0542731086-1.6768649426-2.6434663176$

C - $0.2067362025-1.8312097430-3.7149425358$

C $1.2030730279-1.8507617682-3.5187320884$

C $1.7225668021-1.7094675604-2.2544621384$

C - $2.7764907134-0.7277286852-0.3246145007$

$\begin{array}{lllll}C-2.7764943628 & 0.7277123055 & 0.3246145111\end{array}$

H $2.7971050066-1.7300896292-2.1083010936$

H $1.8626389218-1.9791729374-4.3714283224$

H -0.6115729569 -1.9428099428 - 4.7159720646

$\mathrm{H}-2.1247589753-1.6824345503-2.8108738938$

H $3.5765836595-1.3068691056-0.1536346280$

H $3.0368846894-0.62005876541 .3783954915$

H -2.7970972784 -1.7301085154 2.1083013937

H - 1.8626270318 -1.9791853918 4.3714288292

H o.6115846548 -1.9428062242 4.7159717097

H $2.1247663919-1.68242177442 .8108729781$

$\mathrm{H}-3.0368818082-0.6200760728-1.3783958734$

$\mathrm{H}-3.5765776135 \quad-1.30688971440 .1536345933$

H -2.7971070678 1.7300896364 -2.1083015225

$\mathrm{H}-1.8626383723 \quad 1.9791728273 \quad-4.3714291794$

H o.611573768o 1.9428098383 -4.7159717101

$\begin{array}{llll}\text { H } 2.1247573369 & 1.6824346320 & -2.8108729212\end{array}$

$\mathrm{H}-3.5765847518$ 1.3068694171 -0.1536344192 
$\mathrm{H}-3.03688485190 .6200583645 \quad 1.3783964573$ H 2.7970952292 $1.7301084737 \quad 2.1083009602$ H $1.86262760491 .9791854510 \quad 4.3714279702$ H -o.6115838282 1.94280633124 .7159720719 $\mathrm{H}-2.12476802521 .68242170442 .8108739593$ $\begin{array}{llll}\mathrm{H}_{3} 3.0368816585 & 0.6200765622 & -1.3783949118\end{array}$ H $3.5765764594 \quad 1.30688945790 .1536348344$ 